\title{
Combined Pricing and Portfolio Option Procurement
}

\author{
Qi Fu \\ Industrial Engineering and Logistics Management, Hong Kong University of Science of Technology, \\ Clear Water Bay, Kowloon, Hong Kong, fuqi@ust.hk
}

\author{
Sean X. Zhou \\ Systems Engineering and Engineering Management, The Chinese University of Hong Kong, Shatin, \\ N.T., Hong Kong, zhoux@se.cuhk.edu.hk
}

\author{
Xiuli Chao \\ Industrial and Operations Engineering, University of Michigan, Ann Arbor, Michigan 48109, USA, xchao@umich.edu \\ Chung-Yee Lee \\ Industrial Engineering and Logistics Management, Hong Kong University of Science of Technology, \\ Clear Water Bay, Kowloon, Hong Kong, cylee@ust.hk
}

\begin{abstract}
In this paper, we study a single-product periodic-review inventory system that faces random and price-dependent demand. 1 The firm can purchase the product either from option contracts or from the spot market. Different option contracts are offered by a set of suppliers with a two-part fee structure: a unit reservation cost and a unit exercising cost. The spot market price is random and its realization may affect the subsequent option contract prices. The firm decides the reservation quantity from each supplier and the product selling price at the beginning of each period and the number of options to exercise (inventory replenishment) at the end of the period to maximize the total expected profit over its planning horizon. We show that the optimal inventory replenishment policy is order-up-to type with a sequence of decreasing thresholds. We also investigate the optimal option-reservation policy and the optimal pricing strategy. The optimal reservation quantities and selling price are shown to be both decreasing in the starting inventory level when demand function is additive. Building upon the analytical results, we conduct a numerical study to unveil additional managerial insights. Among other things, we quantify the values of the option contracts and dynamic pricing to the firm and show that they are more significant when the market demand becomes more volatile.
\end{abstract}

Key words: dynamic pricing; portfolio procurement; option contracts; optimal policies

History: Submitted: May 2007; Accepted: February 2011 by Panos Kouvelis, after 3 revisions.

\section{Introduction}

In today's fast changing and highly competitive market environment, product demand has become more whimsical than ever before, and the supply faces both volume and price uncertainties, making it a challenge to match supply with demand. The mismatch can cause companies to suffer from excess inventory and lost sales, which in turn threatens companies' profitability and competitiveness. In this environment, only those companies that can incorporate "change" into their business strategy will have the capability to survive the ruthlessness in this highly competitive era. To add the ingredient of "change" to the recipe of successful coordination of supply and demand, companies can, on the one hand, enhance flexibility of their procurement strategies to manage the supply, and on the other hand, dynamically adjust prices over time to manipulate demand.
On the supply side, using a spectrum of supply alternatives with different flexibility and price combinations allows buyers to spread the risk over suppliers. There are many different contracts provided in the supply market. For example, under the wholesale price contract, buyers commit to suppliers for a fixed quantity by making full payment, before knowing the demand. Thus, the buyers have no flexibility and bear all the inventory risk, while the suppliers enjoy the profit margin without exposure to demand uncertainty. Some risk-sharing contracts have been proposed in the literature, such as buy-back contracts, revenue-sharing contracts, and more recently, option contracts. Although different in format, these contracts shift some risk from buyers to suppliers. For example, option contracts are characterized by a two-part fee structure, a unit cost to reserve options, and an additional unit cost to exercise the options. That is, buyers can reserve the right to buy a certain 
Fu, Zhou, Chao, and Lee: Combined Pricing and Portfolio Option Procurement Production and Operations Management 21(2), pp. 361-377, (c) 2011 Production and Operations Management Society

amount of the product in future at the fixed unit exercising cost by purchasing options now. However, there is no obligation to exercise the options, and buyers can simply let some or all the options elapse if market conditions change later, e.g., demand fails to materialize or market price goes down. With this type of contract, buyers can not only protect themselves against price spikes in future but also enjoy the flexibility of altering the exercising quantity. The supplier, upon signing the option contract, must ensure availability of the product and therefore will take over some inventory risk. Obviously, the sharing of risk depends on the two-part fee structure of option contracts. Besides the contract market, buyers can also buy from the open spot market whenever needed without reservation. However, the spot price is usually quite volatile, depending on the total market supply and demand. Thus the spot market provides buyers with the most flexibility in terms of quantity, yet buyers face a high price risk. Facing the diversified supply schemes, buyers can use a portfolio approach to manage their supply, segmenting demand based on likelihood and fitting each segment with an appropriate supply scheme.

Many industry practices have validated the effectiveness of the portfolio procurement approach in mitigating supply risk and increasing flexibility. HP's Procurement Risk Management program, initiated in year 2000, is basically a portfolio sourcing approach and it has saved the company over US $\$ 425$ million (Nagali et al. 2008). Specifically, HP segments demand into three scenarios: low, medium, and high, based on the risk associated. For low-risk demand, HP commits to the supplier for a fixed quantity to bargain for a better price. For medium-risk demand, option contracts are used to maintain the flexibility of adjusting quantities. And the high-risk demand is left to the spot market. This portfolio approach allows HP to better coordinate supply with demand and the expense of purchasing options is far less than the cost of carrying excess inventory and buying from the spot market with volatile spot price (Bartholomew 2005). The US Department of Water Resources (DWR) has implemented a fuel procurement strategy centered around the use of a balanced fuel portfolio to provide the flexibility to respond to high demand volatility since the energy crisis in July 2000. This approach smooths the impact of uncertainty in demand and supply, and has been proved to be cost effective. ${ }^{1}$ There are also many other examples illustrating the benefits of the portfolio procurement approach.

On the demand side, varying prices over time is often the most natural way to affect customer demand. The intense competition today has made product demand much more sensitive to the price chosen and thus significantly influenced the way that firms price their products. Traditionally, firms focus mainly on their inventory management to cope with demand uncertainty. However, no matter how good inventory management is in reducing supply chain costs, a large portion of retailers still lose millions of dollars annually due to lost sales or excess inventory (Elmaghraby and Keskinocak 2003). Nowadays, many companies adopt dynamic pricing to respond to market fluctuations and uncertainty in demand, because of the simple logic: if the price rises, fewer customers are willing to buy; if the price falls, more customers will be attracted. Therefore "price," acting as an operational tool, can manipulate, boost, or discourage demand in the short run to better balance inventory with demand. Firms use various forms of dynamic pricing, such as markdowns, discounts, and clearance sales. In the last couple of years, we have witnessed an increased application of such dynamic pricing strategies.

Managing supply and demand simultaneously gives companies a greater degree of freedom to cope with volatile market conditions. To understand this strategically, we develop a model to study the strategic decisions of a firm which integrates the portfolio approach in managing the supply with dynamic pricing in managing the demand. Specifically, we consider a finite planning horizon, single item, periodic-review inventory system with price-dependent random demand. The firm uses a portfolio of option contracts ${ }^{2}$ as well as the spot market for the supply of the product. At the beginning of each period, given the initial inventory level, the firm needs to decide the selling price of the product and reserve options from a set of suppliers. Then demand and the spot price are realized and at the end of the period, the firm determines its replenishment quantities, i.e., the quantities of options to be exercised and the quantity to be purchased from the spot market. Unmet demand is fully backlogged. The system incurs inventory holding and backlogging costs. Our objective in this study is to investigate the optimal pricing, option-reservation and exercising strategies to maximize the total profit over the entire planning horizon.

For this model, we show that the optimal inventory replenishment follows an order-up-to policy which is characterized by a sequence of thresholds. That is, there exists a series of target inventory levels, such that if the inventory level after demand realization in a period falls in a specific range then it is optimal to try to order up to the corresponding threshold level of that particular range. The option-reservation quantity is decreasing in both the initial inventory level and the selling price. We further present the optimality conditions that can be used to compute the optimal option-reservation policy. An effective heuristic for near-optimal reservation quantities is also developed. When demand function is additive, we show that the optimal selling price is decreasing in the initial 
inventory level, and thus a list price policy is optimal. Furthermore, we extend the model and results to the case with multi-period option contracts. Based on the analytical results, we conduct a numerical study to reveal additional managerial insights. We quantify the values of the option contracts procurement and dynamic pricing to the firm and show they are more significant when the market demand is more volatile. And we find that the value of dynamic pricing is not very sensitive to the demand variability and the price spread of the reservation prices.

The rest of this paper is organized as follows. In section 2, we provide a review of the related literature. Section 3 presents the model and problem formulation. Section 4 derives the optimal replenishment policy. Section 5 analyzes the optimal option-reservation strategy and provides optimality conditions. Section 6 addresses the dynamic pricing policy. Section 7 extends the model by considering multiperiod option contracts. We conduct numerical studies in section 8 and conclude the paper in section 9 . Throughout the paper, we use "increasing" and "decreasing" in a weak sense, i.e., they represent "nondecreasing" and "nonincreasing," respectively.

\section{Literature Review}

Our work generalizes two streams of research work in the literature. The first one is on the portfolio approach to option procurement. In a single-period setting, Schummer and Vohra (2003) examine the issue of a single buyer who procures options from multiple suppliers. Under the assumption that the shortage cost is arbitrarily high, the total quantity of options procured by the buyer must equal the maximum possible value of demand. They formulate the problem as a network flow problem and propose a class of incentive-compatible, efficient auction mechanisms for procuring options. Their analysis emphasizes the role of the demand distribution. Integrating the contract and spot markets to hedge against risk has received considerable attention recently. See Haksoz and Seshadri (2007) for an extensive review. Here, we review those studies that are closely related to ours. Wu and Kleindorfer (2005) analyze a model in which one buyer procures from multiple suppliers offering option contracts as well as a spot market with random price. They characterize the structure of the optimal portfolio of options and spot market transactions for the buyer and the option pricing issue of the sellers. Fu et al. (2010) consider a single-period portfolio contract procurement problem when demand and spot market price are random and correlated. They analyze the contract selection problem, investigate the effect of correlation and spot market price volatility, and study the portfolio effect. They also de- velop a graphical approach to solve the problem and view the optimal cost function.

In a multi-period setting, $\mathrm{Yi}_{\mathrm{i}}$ and Scheller-Wolf (2003) study an inventory management problem with a finite or an infinite planning horizon. In their model, a buyer who faces random demand has two supply options, a regular supplier and a spot market with random price. Assuming zero lead time, backlogging and a fixed cost of using the spot market, the authors characterize the optimal ordering policy as $(s, S)$ type. Yazlali and Erhun (2004) study a periodic-review finite-horizon dual sourcing problem with two (local and global) suppliers offering the same product with complementary services. They show that a two-level modified base-stock policy is optimal for a wide range of transfer prices. Our paper is most closely related to Martínez-de-Albéniz and Simchi-Levi (2005) who also consider a multi-period portfolio procurement model. The optimal replenishment strategy for a given set of contracts as well as the structure of the optimal contract portfolio from a pool of suppliers are presented. In their model both the composition (which supplier is in) and quantities of the portfolio of contacts are predetermined at the beginning of the planning horizon and fixed over the entire horizon. However, our model allows the firm to dynamically adjust the option-reservation quantities in each period and integrates the selling price decision with optionreservation and exercising decisions.

The second related stream of literature focuses on the joint pricing and inventory decision, where the demand is assumed to be price dependent. There has been a substantial and growing body of literature in this stream of research, starting with Whitin's (1955) seminal paper. In a multi-period environment with uncertain demand and no setup cost, Zabel (1972) studies the case in which the demand consists of an additive random price-dependent part and a deterministic concave demand function. He establishes the existence and uniqueness of the optimal solution by restricting the random part to follow a uniform or exponential distribution. The result is extended by Thowsen (1975) to incorporate more general random terms. The conditions under which the single-period results can be carried over to multiple periods are also discussed in this paper. Federgruen and Heching (1999) consider a model in which the firm periodically reviews inventory and jointly optimizes the price and inventory. They show that a base-stock list price policy is optimal for the model. This model is later extended to include a fixed ordering cost by several researchers under various settings (e.g., Chen and Simchi-Levi 2004a, b, Chen et al. 2006, Feng and Chen 2007, Polatoglu and Sahin 2000). The problem is also studied under a continuous review setting by Feng and Chen (2003), Chao and Zhou (2006), Chen and 
Simchi-Levi (2006), and Chen et al. (2010). Zhu and Thonemann (2009) extend the model of Federgruen and Heching (1999) by considering multiple products. For a more detailed review, readers may refer to several recent survey papers, e.g., Yano and Gilbert (2005), Elmaghraby and Keskinocak (2003), and Chan et al. (2004). Our paper differs from the aforementioned studies in that we consider the portfolio sourcing with multiple supply sources.

\section{The Model}

A firm sells a single product that has uncertain and price-dependent customer demand. For each period $t$, let $D_{t}\left(p_{t}, \varepsilon_{t}\right)$ denote the customer demand with $p_{t}$ being the unit selling price and $\varepsilon_{t}$ a nonnegative random noise term that is i.i.d. across different periods with $\operatorname{cdf} F(\cdot)$ and pdf (or probability mass function) $f(\cdot)$. We assume the demand has either one of the following two functional forms:

$$
\begin{aligned}
& \text { additive form : } D_{t}\left(p_{t}, \varepsilon_{t}\right)=\theta-b p_{t}+\varepsilon_{t}, \\
& \text { multiplicative form : } D_{t}\left(p_{t}, \varepsilon_{t}\right)=\left(\theta-b p_{t}\right) \varepsilon_{t},
\end{aligned}
$$

where $\theta-b p_{t}(\theta>0, b>0$ are constants) captures the price-dependency of demand. Both the additive and multiplicative demand functions are widely adopted in studying joint pricing and inventory decisions (see Petruzzi and Dada (1999) for a review of these two demand models). To ensure nonnegative demand, we assume $p \in[p, \bar{p}]$ for some lower and upper limits $p$ and $\bar{p}$.

The firm adopts a portfolio procurement strategy consisting of a set of $N$ option contracts at various levels of flexibility. With an option contract, say from supplier $i$, the firm needs to purchase options with an upfront unit $\operatorname{cost} C_{t}^{i}$ to reserve a certain number of the product at the beginning of each period $t$, and can exercise the options up to the quantity reserved with an additional unit exercising $\operatorname{cost} e_{t}^{i}$ at the end of period $t$ after the realization of demand. The unit cost $C_{t}^{i}$ is random and is announced by supplier $i$ only at the beginning of period $t$. We index the contracts in increasing order of the exercising costs $e_{t}^{i}$ i.e., $e_{t}^{1}<e_{t}^{2}<\cdots<e_{t}^{N}$. For convenience, let $\bar{e}_{t}=\left(e_{t}^{1}, e_{t}^{2}, \ldots, e_{t}^{N}\right)$. The reservation quantities in each period can be adjusted (the case that an optionreservation decision runs for more than one period is studied in section 7) while the right of exercising the options is valid only for one period. That is, the options will expire if they are not exercised in that period. We assume the realization $c_{t}^{i}$ of $C_{t}^{i}$ is decreasing in $i$ as otherwise, some contracts will be dominated by others and can be disregarded in the period. In addition to the option contracts, the firm can also make immediate purchase through the spot market with price $P_{t}^{s}$, which is random and may be correlated between periods.

There are two common approaches to model the spot market: one is to assume a closed spot market where a few dominant players participate, who also control the contract market (Milner and Kouvelis 2007); the other is to assume an open spot market with a large group of suppliers, a small subset of which operates in the contract market $(\mathrm{Wu}$ and Kleindorfer 2005). For the former case, correlation between demand and the spot price cannot be ignored and the spot market may have only limited supply. But for the latter, it is reasonable to assume an unlimited supply of the spot market as well as independence between the spot price and a single firm's demand, because the spot market price is determined by the total supply and demand of a large number of players and a single firm's demand has a negligible impact on the whole market. Hence, whether correlation between demand and the spot market price should be considered is then a matter of whether one is interested in analyzing a closed or an open market. In this paper, we consider a large open market.

However, the correlation between option contract prices and the spot market price may not be neglected, because information conveyed by price signals, e.g., scarcity or oversupply, can be transmitted across different markets. The spot market price reflects the supply and demand equilibrium, and therefore to some extent, also predicts the option price evolution. It is likely that the suppliers in the contract market, when adjusting option prices, will refer to the realized spot price in the previous periods. Hence, we allow correlation between the option prices and the spot price in our model. It is quite natural that, if the spot market price in the previous period is high, suppliers would tend to set higher option prices for the current period. Specifically, we assume that the optionreservation prices $C_{t}^{i}, i=1, \ldots, N$, evolve stochastically with $p_{t-1}^{s}$, the realized spot price in the previous period. We model $\left\{P_{t}^{s}, 0 \leq t \leq T\right\}$ as a discrete time Markov chain (the distribution of $P_{t}^{s}$ only depends on the realization of $P_{t-1}^{s}$ ) with $P_{0}^{s}$ a degenerate random variable and independent of the option and exercising prices set by the suppliers and the purchasing decisions of the firm. Consequently, the option prices $C_{t}^{i}$ are Markov modulated. We assume the firm has some knowledge about $C_{t}^{i}$ in the form of its distribution function after observing $P_{t-1}^{s}=p_{t-1}^{s}$. As there are multiple random variables involved in the firm's decision, we will denote the mathematical expectation by $E_{Z}$ when the expectation is taken with respect to a random variable $Z$.

At the beginning of each period, the reservation prices from the suppliers are realized based on the spot price in the previous period. Then after 
reviewing the inventory status, the firm decides the selling price and then purchases options from the suppliers to ensure certain quantities of the product available for the current period; then demand and the spot market price are realized; and finally, the inventory replenishment decision is made at the end of the period. Any unsatisfied customer demands are backlogged. We assume that the backlogged customers will pay the price in the period when the demand was made. This is a common assumption in joint inventory and pricing literature, see, e.g., Federgruen and Heching (1999), Chen and SimchiLevi $(2004 a, b)$. In practice, if the price goes down in a subsequent period, then customers may sometimes demand a refund for the difference in price, which we do not consider in this paper.

For $i=1, \ldots, N$, let $a_{t}^{i}$ be the total number of options purchased from suppliers 1 through $i$ at the beginning of period $t$ and $a_{t}^{0}=0$. Then $a_{t}^{i}-a_{t}^{i-1}$ is the reservation quantity from supplier $i$. Let $\bar{a}_{t}=\left(a_{t}^{0}, a_{t}^{1}, a_{t}^{2}, \ldots, a_{t}^{N}\right)$ be the option-reservation vector. Then in period $t$ with given option prices $C_{t}^{i}=c_{t}^{i}$, the total option-reservation cost is

$$
\sum_{i=1}^{N} c_{t}^{i}\left(a_{t}^{i}-a_{t}^{i-1}\right)
$$

For each period $t$, we define

$x_{t}=$ the starting inventory level,

$x_{t}^{\prime}=$ the inventory level after demand realization

but before replenishment,

$y_{t}=$ the inventory level after replenishment.

Given the inventory level after demand realization $x_{t}^{\prime}$ and the target inventory level $y_{t}$, the firm will exercise only those options with exercising cost less than the current (realized) spot price in increasing order of their indices (recall that the suppliers are indexed in increasing order of their exercising costs) until they are depleted. After that, if necessary, the firm will order some from the spot market. It is clear that $y_{t}-x_{t}^{\prime}$ represents the total ordering quantity of the firm in period $t$. If $y_{t}-x_{t}^{\prime}$ is less than $a_{t}^{1}$, the reserved quantity from supplier 1 , then the total exercising cost is $e_{t}^{1}\left(y_{t}-x_{t}^{\prime}\right)$; if $y_{t}-x_{t}^{\prime}$ is more than $a_{t}^{1}$ but less than the total reserved quantity $a_{t}^{2}$ from suppliers 1 and 2, then the exercising cost is

$$
\begin{aligned}
e_{t}^{1} a_{t}^{1}+e_{t}^{2}\left(y_{t}-x_{t}^{\prime}-a_{t}^{1}\right)= & e_{t}^{1}\left(y_{t}-x_{t}^{\prime}\right)+\left(e_{t}^{2}-e_{t}^{1}\right) \\
& \times\left(y_{t}-x_{t}^{\prime}-a_{t}^{1}\right),
\end{aligned}
$$

where the second term on the right-hand side of the equality can be considered as the additional cost incurred by ordering from supplier 2. In general, for a particular realization of the spot price $P_{t}^{s}=p_{t}^{s}$, the inventory replenishment cost function with a given ordering quantity $q$ and reservation quantities $\bar{a}_{t}$ can be written as, letting $e_{t}^{0}=0$,

$\mathscr{C}_{t}^{E}\left(q, \bar{a}_{t}, p_{t}^{s}\right)$
$= \begin{cases}e_{t}^{1} q+\cdots+\left(e_{t}^{i}-e_{t}^{i-1}\right)\left(q-a_{t}^{i-1}\right)^{+}+\left(p_{t}^{s}-e_{t}^{i}\right)\left(q-a_{t}^{i}\right)^{+}, & \text {if } i>0, \\ p_{t}^{s} q, & \text { if } i=0,\end{cases}$

where $i=\arg \max _{0 \leq j \leq N}\left\{e_{t}^{j}<p_{t}^{s}\right\}$ and $x^{+}=\max \{x, 0\}$. Note that it is possible that some suppliers may turn out to set their exercising prices higher than the spot market price in period $t$, because when setting the prices at the beginning of the period, the spot market price is still uncertain. Therefore the number of option contracts exercised depends on the realization of the spot market price. The function $\mathscr{C}_{t}^{E}\left(q, \bar{a}_{t}, p_{t}^{s}\right)$ is piecewise linear convex in $q$.

We denote by $G_{t}(y)$ the one-period inventory holding and backlogging costs and assume the following property.

Assumption 1. $G_{t}(\cdot)$ is a convex function and $\lim _{|y| \rightarrow \infty} G_{t}(y)=\infty$.

Our objective is to analyze the selling price, portfolio option-reservation and inventory replenishment decisions in each period so that the total expected profit of the firm over a finite planning horizon with length $T$ is maximized.

Let $V_{t}\left(x_{t}, p_{t-1}^{s}\right)$ be the maximum total expected profit from the beginning of period $t$ until the end of the planning horizon given that the initial inventory level is $x_{t}$ and the spot market price is $p_{t-1}^{s}$ in period $t-1$. The dynamic program can be formulated as

$$
\begin{aligned}
V_{t}\left(x_{t}, p_{t-1}^{s}\right)= & \mathrm{E}_{C_{t}}\left[\operatorname { m a x } _ { p _ { t } \in [ \underline { p } , \overline { p } ] } \left\{p_{t} \mathrm{E}_{\varepsilon_{t}}\left[D_{t}\left(p_{t}, \varepsilon_{t}\right)\right]\right.\right. \\
& +\max _{a_{t}^{N} \geq \cdots \geq a_{t}^{1} \geq 0}\left(-\sum_{i=1}^{N} C_{t}^{i}\left(a_{t}^{i}-a_{t}^{i-1}\right)\right. \\
& \left.\left.\left.+\mathrm{E}_{\varepsilon_{t}, P_{t}^{s}}\left[U_{t}\left(x_{t}-D_{t}\left(p_{t}, \varepsilon_{t}\right), \bar{a}_{t}, P_{t}^{s}\right)\right]\right)\right\}\right]
\end{aligned}
$$

where the first term within the curly brackets represents the firm's one-period expected revenue from selling the product, the second term (the first term within the parentheses) is the option-reservation cost in period $t$, and the last term, the expected optimal future profit function, accounts for the inventory replenishment cost, the one-period inventory holding and backlogging cost, and the expected future profit. That is,

$$
\begin{aligned}
U_{t}\left(x_{t}^{\prime}, \bar{a}_{t}, P_{t}^{s}\right)= & \max _{y \geq x_{t}^{\prime}=x_{t}-D_{t}\left(p_{t}, \varepsilon_{t}\right)}\left\{-\mathscr{C}_{t}^{E}\left(y-x_{t}^{\prime}, \bar{a}_{t}, P_{t}^{s}\right)\right. \\
& \left.-G_{t}(y)+V_{t+1}\left(y, P_{t}^{s}\right)\right\} .
\end{aligned}
$$

It should be noted that, in Equation (1) and wherever applicable in the following: $\mathrm{E}_{C_{t}}[\cdot]:=\mathrm{E}_{C_{t}}\left[\cdot \mid P_{t-1}^{s}=p_{t-1}^{s}\right]$ 
and $\mathrm{E}_{P_{t}^{s}}[\cdot]:=\mathrm{E}_{P_{t}^{s}}\left[\cdot \mid P_{t-1}^{s}=p_{t-1}^{s}\right]$. The terminal condition is $V_{T+1}\left(x, p_{T}^{s}\right) \equiv 0$.

In the subsequent analysis, we use $p_{t}^{s}$ and $c_{t}^{i}$ to represent a particular sample path of $P_{t}^{s}$ and $C_{t}^{i}$, respectively.

\section{Inventory Replenishment Policy}

In this section, we characterize the structure of the optimal inventory replenishment policy. Recall that the firm decides the inventory replenishment by exercising options or purchasing from the spot market after demand and the spot market price are realized.

We first prove that $U_{t}\left(x, \bar{a}, p_{t}^{s}\right)$ and $V_{t}\left(x, p_{t-1}^{s}\right)$ are concave functions. For notational simplicity, we skip the subscript $t$ unless confusion may arise.

THEOREM 1. For $t=1, \ldots, T$,

(a) $U_{t}\left(x^{\prime}, \bar{a}, p_{t}^{s}\right)$ is concave in $\left(x^{\prime}, \bar{a}\right)$ and increasing in $\bar{a}$,

(b) $V_{t}\left(x, p_{t-1}^{s}\right)$ is concave in $x$.

Proof. We prove the results by induction on $t$. When $t=T$, as $-\mathscr{C}_{T}^{E}\left(y-x^{\prime}, \bar{a}, p_{T}^{s}\right)$ is jointly concave in $\left(x^{\prime}, y, \bar{a}\right)$ and $-G_{T}(y)$ is concave in $y$, from the boundary condition $V_{T+1}\left(x, p_{T}^{s}\right)=0$ and

$$
U_{T}\left(x^{\prime}, \bar{a}, p_{T}^{s}\right)=\max _{y \geq x^{\prime}}\left\{-\mathscr{C}_{T}^{E}\left(y-x^{\prime}, \bar{a}, p_{T}^{s}\right)-G_{T}(y)\right\},
$$

$U_{T}\left(x^{\prime}, \bar{a}, p_{T}^{s}\right)$ is jointly concave in $\left(x^{\prime}, \bar{a}\right)$ following from Proposition B-4 in Heyman and Sobel (1984). Thus, with analogous arguments,

$\max _{a^{N} \geq a^{N-1} \geq \cdots \geq a^{1} \geq 0}\left\{-\sum_{i=1}^{N} c_{t}^{i}\left(a^{i}-a^{i-1}\right)+\mathrm{E}_{P_{T}^{s}, \varepsilon_{T}}\left[U_{T}\left(x^{\prime}, \bar{a}, P_{T}^{s}\right)\right]\right\}$

is concave in $x^{\prime}$ and thus jointly concave in $(x, p)$ because $x^{\prime}=x-D_{T}\left(p, \varepsilon_{T}\right)$ and $D_{T}\left(p, \varepsilon_{T}\right)$ is linear in $p$. Therefore, $V_{T}\left(x, p_{T-1}^{s}\right)$ is concave in $x$ by preservation of concavity after maximization and expectation. Now suppose $V_{t}\left(x, p_{t-1}^{s}\right)$ is concave in $x$ for some $t, 2 \leq$ $t \leq T$. Concavity of $U_{t-1}\left(x^{\prime}, \bar{a}, p_{t-1}^{s}\right)$ and $V_{t-1}\left(x, p_{t-2}^{s}\right)$ can also be easily verified because for any realization $c_{t}^{i}$ and $p_{t-1}^{s}$ every term of either function is concave, and concavity is preserved after expectation and maximization operations. So we complete the proof of concavity.

Finally, as $\mathscr{C}_{t}^{E}\left(\cdot, \bar{a}, p_{t-1}^{s}\right)$ is decreasing in $\bar{a}, U_{t}\left(x^{\prime}, \bar{a}, p_{t}^{s}\right)$ is increasing in $\bar{a}$.

The monotonic property in $\bar{a}$ of $U_{t}\left(x^{\prime}, \bar{a}, p_{t}^{s}\right)$ is intuitive and can be explained as follows. With a higher given option-reservation level, the potential replenishment cost will be lower due to the lower likelihood of exercising or purchasing at a higher cost, which in turn results in higher future profit.

The optimal replenishment policy for each period is given in the following theorem.
THEOREM 2. For $t=1, \ldots, T$, given the inventory level after demand realization in period $t$ is $x^{\prime}$, the options available for exercising $\bar{a}_{t}=\left(a_{t}^{0}, a_{t}^{1}, a_{t}^{2}, \ldots, a_{t}^{N}\right)$, and the realized spot market price $P_{t}^{s}=p_{t}^{s}$, if $i=\arg \max _{0 \leq j \leq N}\left\{e_{t}^{j}<p_{t}^{s}\right\}>0$, then there exist a sequence of thresholds $S_{t}^{1}\left(p_{t}^{s}\right)>S_{t}^{2}\left(p_{t}^{s}\right)$ $>\cdots>S_{t}^{i}\left(p_{t}^{s}\right)>\tilde{S}_{t}\left(p_{t}^{s}\right)$, such that the optimal target inventory level $y_{t}^{*}$ is given by

$$
y_{t}^{*}= \begin{cases}x^{\prime} & \text { if } x^{\prime} \geq S_{t}^{1}\left(p_{t}^{s}\right), \\ S_{t}^{1}\left(p_{t}^{s}\right) & \text { if } S_{t}^{1}\left(p_{t}^{s}\right)>x^{\prime} \geq S_{t}^{1}\left(p_{t}^{s}\right)-a_{t}^{1}, \\ x^{\prime}+a_{t}^{1} & \text { if } S_{t}^{1}\left(p_{t}^{s}\right)-a_{t}^{1}>x^{\prime} \geq S_{t}^{2}\left(p_{t}^{s}\right)-a_{t}^{1}, \\ S_{t}^{2}\left(p_{t}^{s}\right) & \text { if } S_{t}^{2}\left(p_{t}^{s}\right)-a_{t}^{1}>x^{\prime} \geq S_{t}^{2}\left(p_{t}^{s}\right)-a_{t}^{2}, \\ x^{\prime}+a_{t}^{2} & \text { if } S_{t}^{2}\left(p_{t}^{s}\right)-a_{t}^{2}>x^{\prime} \geq S_{t}^{3}\left(p_{t}^{s}\right)-a_{t}^{2}, \\ \vdots & \\ S_{t}^{i}\left(p_{t}^{s}\right) & \text { if } S_{t}^{i}\left(p_{t}^{s}\right)-a_{t}^{i-1}>x^{\prime} \geq S_{t}^{i}\left(p_{t}^{s}\right)-a_{t}^{i}, \\ x^{\prime}+a_{t}^{i} & \text { if } S_{t}^{i}\left(p_{t}^{s}\right)-a_{t}^{i}>x^{\prime} \geq \tilde{S}_{t}\left(p_{t}^{s}\right)-a_{t}^{i}, \\ \tilde{S}_{t}\left(p_{t}^{s}\right) & \text { if } x^{\prime}<\tilde{S}_{t}\left(p_{t}^{s}\right)-a_{t}^{i},\end{cases}
$$

where $S_{t}^{j}\left(p_{t}^{s}\right) \in \arg \max _{y}\left\{-e_{t}^{j} y-G_{t}(y)+V_{t+1}\left(y, p_{t}^{s}\right)\right\}$ for $j=1, \ldots, i$, and $\tilde{S}_{t}\left(p_{t}^{s}\right)=\arg \max _{y}\left\{-p_{t}^{s} y-G_{t}(y)+V_{t+1}\right.$ $\left.\left(y, p_{t}^{s}\right)\right\}$. If $i=0$, it is optimal to order up to $\tilde{S}_{t}\left(p_{t}^{s}\right)$ when $x^{\prime}<\tilde{S}_{t}\left(p_{t}^{s}\right)$ and order nothing otherwise.

Proof. The theorem is proved by using the concavity of $-G_{t}(y)+V_{t+1}\left(y, p_{t}^{s}\right)$ and the piecewise linear convex structure of the exercising cost function $\mathscr{C}_{t}^{E}\left(y-x^{\prime}, \bar{a}_{t}, p_{t}^{s}\right)$. First note that, because $e_{t}^{1}<e_{t}^{2}<\cdots$ $<e_{t}^{i}<p_{t}^{s}, \quad S_{t}^{1}\left(p_{t}^{s}\right)>S_{t}^{2}\left(p_{t}^{s}\right)>\cdots>S_{t}^{i}\left(p_{t}^{s}\right)>\tilde{S}_{t}\left(p_{t}^{s}\right) \quad$ from their definition.

If $i>0$, i.e., there exists at least one option contract with exercising cost lower than the realized spot price, the exercising cost function has at least two segments and we have the following four cases:

1. $x^{\prime} \geq S_{t}^{1}\left(p_{t}^{s}\right)$.

In this case, for $y \geq x^{\prime}$, the derivative of function $-G_{t}(y)+V_{t+1}\left(y, p_{t}^{s}\right)$ is less than $e_{t}^{1}$ and is decreasing in $y$ by definition of $S_{t}^{1}\left(p_{t}^{s}\right)$ and the concavity of $-G_{t}(y)+V_{t+1}\left(y, p_{t}^{s}\right)$. However, the unit exercising cost is at least $e_{t}^{1}$. This implies that the cost of exercising one more unit is higher than the marginal revenue of this unit, so it is optimal to order nothing and keep inventory at the level of $x^{\prime}$.

2. $S_{t}^{j}\left(p_{t}^{s}\right)-a_{t}^{j} \leq x^{\prime}<S_{t}^{j}\left(p_{t}^{s}\right)-a_{t}^{j-1}$ for $j=1, \ldots, i$.

In this case, we have $x^{\prime}+a_{t}^{j-1}<S_{t}^{j}\left(p_{t}^{s}\right) \leq x^{\prime}+a_{t}^{j}$. For $x^{\prime}<y<S_{t}^{j}\left(p_{t}^{s}\right)$, the gain of exercising one unit is greater than the maximum unit exercising cost $e_{t}^{j}$, so the objective function is increasing in $y$. On the other hand, for $y>S_{t}^{j}\left(p_{t}^{s}\right)$, the objective value is decreasing in $y$, as the marginal increase of 
revenue is less than the unit exercising cost. Thus $y_{t}^{*}=S_{t}^{j}\left(p_{t}^{s}\right)$.

3. $S_{t}^{j+1}\left(p_{t}^{s}\right)-a_{t}^{j}\left(\tilde{S}_{t}\left(p_{t}^{s}\right)-a_{t}^{i}\right.$ if $\left.j=i\right) \leq x^{\prime}<S_{t}^{j}\left(p_{t}^{s}\right)-a_{t}^{j}$ for $j=1, \ldots, i$.

Then $S_{t}^{j+1}\left(p_{t}^{s}\right) \leq x^{\prime}+a_{t}^{j}<S_{t}^{j}\left(p_{t}^{s}\right)$. Similar to case (2), we can show that the profit function is increasing on $x^{\prime}<y \leq x^{\prime}+a_{t}^{j}$, decreasing on $y \geq$ $x^{\prime}+a_{t}^{j}$ and reaches maximum when $y_{t}^{*}=x^{\prime}+a_{t}^{j}$.

4. $x^{\prime}<\tilde{S}_{t}\left(p_{t}^{s}\right)-a_{t}^{i}$.

Similar to case (2), the profit function is increasing for $x^{\prime}<y<\tilde{S}_{t}\left(p_{t}^{s}\right)$ and decreasing for $y>\tilde{S}_{t}\left(p_{t}^{s}\right)$. Hence $y_{t}^{*}=\tilde{S}_{t}\left(p_{t}^{s}\right)$.

If $i=0$, then the firm will not exercise any options and will replenish solely from the spot market. The replenishment cost function becomes linear and therefore the policy reduces to a base-stock policy.

Theorem 2 shows that the optimal inventory replenishment policy is of order-up-to type and is composed of a sequence of control parameters depending on the realization of the spot market price. This policy results from the piecewise linear convex increasing replenishment cost function and the concave profit function. The number of the threshold values increases with the revealed spot price, since the realized spot price partitions the reserved options into two categories. The first category has exercising cost lower than the spot price, which will be exercised sequentially. The remaining options constitute the second category, which will not be exercised since their exercising costs are higher than the realized spot market price. The higher the spot price, the more types of options in the first category. If the realized spot price is lower than the smallest unit exercising cost, then the firm will let all the reserved options elapse and simply buy from the spot market. In this case, the optimal replenishment policy is just a basestock policy.

REMARK 1. It is noteworthy to point out that when the option prices do not depend on the realized spot market price in the previous period and the spot prices in different periods are independent, then all the thresholds in the optimal inventory replenishment policy, except the last one, will become state-independent. That is, they will not depend on $p_{t}^{s}$ and $S_{t}^{i}\left(p_{t}^{s}\right)$ can be simply written as $S_{t}^{i}$.

The results in this section set the stage for studying the optimal option-reservation policy in the next section.

\section{Option-Reservation Policy}

Reserving options can protect the firm from the risk of sharp price increase in the spot market, yet over- reservation might be costly because of the sunk reservation cost. Thus the optimal reservation policy should balance these risks. As the firm reserves options after the suppliers set the option contract prices, to maintain a flexible procurement strategy the buyer can adjust the option-reservation quantities in each period in response to the changes of on-hand inventory and the selling price. In this section, we first present structural results of the optimal optionreservation policy. Then, based on the optimal replenishment policy characterized in section 4 , we derive a system of optimality equations that determines the reservation quantity of each contract.

LEMMA 1. If $g(\cdot)$ is a concave function and $\beta, \gamma$ nonnegative, then $g(\beta x+\gamma p)$ is submodular in $(x, p)$.

This lemma follows directly from the definitions of concavity and submodularity, and it leads to the following important property of the value function (2).

Lemma 2. For $t=1, \ldots, T, U_{t}\left(x^{\prime}, \bar{a}, p_{t}^{s}\right)$ is a submodular function for each pair of $x^{\prime}$ and $a^{i}$, for $i=1,2, \ldots, N$.

Proof. To show this result, it is equivalent to show that $\tilde{U}_{t}\left(\tilde{x}, \bar{a}, p_{t}^{s}\right) \equiv U_{t}\left(-x^{\prime}, \bar{a}, p_{t}^{s}\right)$ is supermodular in each pair of $\tilde{x}$ and $a^{i}$. To that end, let $q=y-x^{\prime}=y+\tilde{x}$, then

$$
\begin{aligned}
\tilde{U}_{t}\left(\tilde{x}, \bar{a}, p_{t}^{s}\right)= & \max _{q \geq 0}\left\{-\mathscr{C}_{t}^{E}\left(q, \bar{a}_{t}, p_{t}^{s}\right)-G_{t}(q-\tilde{x})\right. \\
& \left.+V_{t+1}\left(q-\tilde{x}, p_{t}^{s}\right)\right\} .
\end{aligned}
$$

Note that, as $V_{t+1}\left(\cdot, p_{t}^{s}\right)$ is concave, $V_{t+1}\left(q-\tilde{x}, p_{t}^{s}\right)$ is supermodular in $q$ and $\tilde{x}$. Similarly, as $G_{t}(\cdot)$ is convex, it follows from Lemma 1 that $-G_{t}(q-\tilde{x})$ is supermodular in $q$ and $\tilde{x}$. Finally, from the definition of $\mathscr{C}_{t}^{E}$, it is also supermodular in $q$ and $a^{i}$. Therefore, as $q \geq 0$ is clearly a lattice and supermodularity is preserved under maximization (Topkis 1998), $\tilde{U}_{t}\left(\tilde{x}, \bar{a}, p_{t}^{s}\right)$ is supermodular in $\left(\tilde{x}, a^{i}\right)$ and $U_{t}\left(x^{\prime}, \bar{a}, p_{t}^{s}\right)$ is a submodular function for each pair of $x^{\prime}$ and $a^{i}$.

Define

$$
\begin{aligned}
J_{t}\left(x, p, \bar{a}, p_{t-1}^{s}\right)= & -\sum_{i=1}^{N} c_{t}^{i}\left(a^{i}-a^{i-1}\right) \\
& +\mathrm{E}_{\varepsilon_{t}, P_{t}^{s}}\left[U_{t}\left(x-D_{t}\left(p, \varepsilon_{t}\right), \bar{a}, P_{t}^{s}\right)\right],
\end{aligned}
$$

and let $\bar{a}_{t}\left(x, p, p_{t-1}^{s}\right) \in \arg \max _{a^{N} \geq a^{N-1} \geq \cdots \geq a^{1} \geq 0}\left\{J_{t}(x, p\right.$, $\left.\left.\bar{a}, p_{t-1}^{s}\right)\right\}$. $J_{t}$ depends on $p_{t-1}^{s}$ since $\mathrm{E}_{\varepsilon_{t}, p_{t}^{s}}[\cdot]$ conditions on $p_{t-1}^{s}$ as we noted previously.

The structural property of the optimal option-reservation quantity $\bar{a}_{t}\left(x, p, p_{t-1}^{s}\right)$ with given inventory level $x$ and price $p$ is stated in the following theorem.

Theorem 3. For $t=1, \ldots$, T and $i=1,2, \ldots, N$,

(a) $J_{t}\left(x, p, \bar{a}, p_{t-1}^{s}\right)$ is submodular for each pair of $\left(x, a^{i}\right)$; 
Fu, Zhou, Chao, and Lee: Combined Pricing and Portfolio Option Procurement Production and Operations Management 21(2), pp. 361-377, (c) 2011 Production and Operations Management Society

(b) $J_{t}\left(x, p, \bar{a}, p_{t-1}^{s}\right)$ is submodular for each pair of $\left(p, a^{i}\right)$;

(c) the optimal reservation quantity $a_{t}^{i}\left(x, p, p_{t-1}^{s}\right)$ is decreasing in $x$ and $p$.

PROOF. The first term of $J_{t}\left(x, p, \bar{a}, p_{t-1}^{s}\right)$ depends only on $\bar{a}$ and thus is trivially submodular in $\left(x, a^{i}\right)$. For the second term, $U_{t}\left(\cdot, \cdot, p_{t}^{s}\right)$ is submodular and concave in its first two components by Lemma 2 . As the expected demand is linear in $p$, it is not hard to see that $U_{t}\left(x-D_{t}\left(p, \varepsilon_{t}\right)\right.$, $\left.\bar{a}, p_{t}^{s}\right)$ is submodular in $\left(x, a^{i}\right)$ and $\left(p, a^{i}\right)$ for both additive and multiplicative demand. Since submodularity is preserved under expectation, parts (a) and (b) follow.

Part (c) follows directly from the submodularity of $J_{t}\left(x, p, \bar{a}, p_{t-1}^{s}\right)$ (Topkis 1998).

The theorem above indicates how the option-reservation quantities change with the starting inventory level and the selling price in each period. It is intuitive as more on-hand stock implies that less future capacity will be needed. The option-reservation quantity also decreases with the selling price, because the demand is decreasing in the selling price $p$, which in turn results in smaller option quantity to be reserved.

The next theorem presents the set of optimality conditions that can be used to solve the optimal $\bar{a}_{t}$. Its proof is given in the Appendix. Let $\mathbf{1}(A)=1$ if $A$ is true; otherwise $\mathbf{1}(A)=0$.

THEOREM 4. Let $R_{t}\left(y, p_{t}^{s}\right)=-G_{t}(y)+V_{t+1}\left(y, p_{t}^{s}\right)$ and let $\bar{F}(\cdot)$ be the complement cumulative distribution function of $\varepsilon_{t}$.

(a) For the additive demand, let $z=x-\theta+b p$. Then the optimal cumulative option-reservation quantities $a_{t}^{i}(x, p$, $\left.p_{t-1}^{s}\right), i=1, \ldots, N-1$ are the solutions of the following optimality conditions,

$$
\begin{aligned}
c_{t}^{i}-c_{t}^{i+1}= & \lambda_{i}-\lambda_{i+1}+\mathrm{E}_{P_{t}^{s}}\left[\mathbf{1}\left(P_{t}^{s} \geq e_{t}^{i+1}\right)\right. \\
& \times\left(\int_{z-S_{t}^{i}\left(P_{t}^{s}\right)+a_{t}^{i}}^{z-S_{t}^{i+1}\left(P_{t}^{s}\right)+a_{t}^{i}}\left[-e_{t}^{i}+R_{t}^{\prime}\left(z-\xi+a_{t}^{i}, P_{t}^{s}\right)\right] f(\xi) d \xi\right. \\
& \left.\left.+\left(e_{t}^{i+1}-e_{t}^{i}\right) \bar{F}\left(z-S_{t}^{i+1}\left(P_{t}^{s}\right)+a_{t}^{i}\right)\right)\right] \\
& +\mathrm{E}_{P_{t}^{s}}\left[\mathbf{1}\left(e_{t}^{i} \leq P_{t}^{s}<e_{t}^{i+1}\right)\right. \\
& \times\left(\int_{z-S_{t}^{i}\left(P_{t}^{s}\right)+a_{t}^{i}}^{z-\tilde{S}_{t}\left(P_{t}^{s}\right)+a_{t}^{i}}\left[-e_{t}^{i}+R_{t}^{\prime}\left(z-\xi+a_{t}^{i}, P_{t}^{s}\right)\right] f(\xi) d \xi\right. \\
& \left.\left.+\left(P_{t}^{s}-e_{t}^{i}\right) \bar{F}\left(z-\tilde{S}_{t}\left(P_{t}^{s}\right)+a_{t}^{i}\right)\right)\right]
\end{aligned}
$$

and for $a_{t}^{N}\left(x, p, p_{t-1}^{s}\right)$, it satisfies

$$
\begin{aligned}
c_{t}^{N}= & \lambda_{N}+\mathrm{E}_{P_{t}^{s}}\left[\mathbf { 1 } ( e _ { t } ^ { N } \leq P _ { t } ^ { s } ) \left(\int_{z-S_{t}^{N}\left(P_{t}^{s}\right)+a_{t}^{N}}^{z-\tilde{S}_{t}\left(P_{t}^{s}\right)+a_{t}^{N}}\right.\right. \\
& {\left[-e_{t}^{N}+R_{t}^{\prime}\left(z-\xi+a_{t}^{N}, P_{t}^{s}\right)\right] f(\xi) d \xi } \\
& \left.\left.+\left(P_{t}^{s}-e_{t}^{N}\right) \bar{F}\left(z-\tilde{S}_{t}\left(P_{t}^{s}\right)+a_{t}^{N}\right)\right)\right]
\end{aligned}
$$

where $\lambda_{i}, i=1, \ldots, N$, satisfies $\lambda_{i}\left(a_{t}^{i}-a_{t}^{i-1}\right)=0$ and $\lambda_{i} \geq 0$.

(b) For the multiplicative demand, let $z(\xi)=$ $x-(\theta-b p) \xi$. Then the optimal cumulative option-reservation quantities $a_{t}^{i}\left(x, p, p_{t-1}^{s}\right), i=1, \ldots, N-1$ are the solutions of the following optimality conditions,

$$
\begin{aligned}
c_{t}^{i}-c_{t}^{i+1}= & \lambda_{i}-\lambda_{i+1}+\mathrm{E}_{P_{t}^{s}}\left[\mathbf { 1 } ( P _ { t } ^ { s } \geq e _ { t } ^ { i + 1 } ) \left(\int_{\frac{x-S_{t}^{i}\left(P_{t}^{s}\right)+a_{t}^{i}}{\theta-b p}}^{\frac{x-S_{t}^{i+1}\left(P_{t}^{s}\right)+a_{t}^{i}}{i}}\right.\right. \\
& {\left[-e_{t}^{i}+R_{t}^{\prime}\left(z(\xi)+a_{t}^{i}, P_{t}^{s}\right)\right] f(\xi) d \xi } \\
& \left.\left.+\left(e_{t}^{i+1}-e_{t}^{i}\right) \bar{F}\left(\frac{x-S_{t}^{i+1}\left(P_{t}^{s}\right)+a_{t}^{i}}{\theta-b p}\right)\right)\right] \\
& +\mathrm{E}_{P_{t}^{s}}\left[\mathbf { 1 } ( e _ { t } ^ { i } \leq P _ { t } ^ { s } < e _ { t } ^ { i + 1 } ) \left(\int_{\frac{x-S_{t}^{i}\left(P_{t}^{s}\right)+a_{t}^{i}}{\theta-b p}}^{\frac{x-\tilde{S}_{t}\left(P_{t}^{s}\right)+a_{t}^{i}}{i}}\right.\right. \\
& {\left[-e_{t}^{i}+R_{t}^{\prime}\left(z(\xi)+a_{t}^{i}, P_{t}^{s}\right)\right] f(\xi) d \xi } \\
& \left.\left.+\left(P_{t}^{s}-e_{t}^{i}\right) \bar{F}\left(\frac{x-\tilde{S}_{t}\left(P_{t}^{s}\right)+a_{t}^{i}}{\theta-b p}\right)\right)\right],
\end{aligned}
$$

and for $a_{t}^{N}\left(x, p, p_{t-1}^{s}\right)$, it satisfies

$$
\begin{aligned}
c_{t}^{N}= & \lambda_{N}+\mathrm{E}_{P_{t}^{s}}\left[\mathbf { 1 } ( e _ { t } ^ { N } \leq P _ { t } ^ { s } ) \left(\int_{\frac{x-S_{t}^{N}\left(P_{t}^{s}\right)+a_{t}^{N}}{\theta-b p}}^{\frac{x-\tilde{S}_{t}\left(P_{t}^{s}\right)+a_{t}^{N}}{2}}\right.\right. \\
& {\left[-e_{t}^{N}+R_{t}^{\prime}\left(z(\xi)+a_{t}^{N}, P_{t}^{s}\right)\right] f(\xi) d \xi } \\
& \left.\left.+\left(P_{t}^{s}-e_{t}^{N}\right) \bar{F}\left(\frac{x-\tilde{S}_{t}\left(P_{t}^{s}\right)+a_{t}^{N}}{\theta-b p}\right)\right)\right],
\end{aligned}
$$

where $\lambda_{i}, i=1, \ldots, N$, satisfies $\lambda_{i}\left(a_{t}^{i}-a_{t}^{i-1}\right)=0$ and $\lambda_{i} \geq 0$.

In the theorem above, $\lambda_{i}$ represents the Lagrange multiplier corresponding to constraint $a^{i} \leq a^{i+1}$. The theorem provides a recursive numerical method for computing the optimal reservation quantities. Since the profit function is concave, we can solve the optimal option-reservation quantities using line search by applying the preceding series of optimality conditions. Because of the computational complexity in solving these equations, in section 8 we shall develop a simple heuristic method to compute the near-optimal reservation quantities.

\section{Dynamic Pricing Policy}

At the beginning of each period, after reviewing the onhand inventory level, the firm adjusts the product selling price to better coordinate supply with demand. In this section we analyze the optimal pricing decision.

Let $K_{t}\left(x, p, p_{t-1}^{s}\right)=p \mathrm{E}_{\varepsilon_{t}}\left[D_{t}\left(p, \varepsilon_{t}\right)\right]+J_{t}\left(x, p, \bar{a}_{t}\left(x, p, p_{t-1}^{s}\right)\right.$, $\left.p_{t-1}^{s}\right)$ and $p_{t}^{*}\left(x, p_{t-1}^{s}\right) \in \arg \max _{p}\left\{K_{t}\left(x, p, p_{t-1}^{s}\right)\right\}$. Apply- 
ing Lemma 1, we can obtain the behavior of the optimal selling price $p_{t}^{*}\left(x, p_{t-1}^{s}\right)$ with respect to the starting inventory level when the demand function is additive.

\section{THEOREM 5. For $t=1, \ldots, T$, if demand is additive, then}

(a) $K_{t}\left(x, p, p_{t-1}^{s}\right)$ is submodular in $(x, p)$;

(b) the optimal selling price $p_{t}^{*}\left(x, p_{t-1}^{s}\right)$ is decreasing in $x$;

(c) the optimal reservation quantity $a_{t}^{i *}\left(x, p_{t-1}^{s}\right)=a_{t}^{i}(x$, $\left.p_{t}^{*}\left(x, p_{t-1}^{s}\right), p_{t-1}^{s}\right)$ is decreasing in $x$.

PROOF. For part (a), as the first term in $K_{t}\left(x, p, p_{t-1}^{s}\right)$ depends only on $p$ which is trivially submodular, it is sufficient to show $J_{t}\left(x, p, \bar{a}_{t}\left(x, p, p_{t-1}^{s}\right), p_{t-1}^{s}\right)$ is submodular. Recall that $J_{t}\left(x, p, \bar{a}_{t}, p_{t-1}^{s}\right)$ is jointly concave in $(x, p, \bar{a})$, so is $J_{t}\left(x, p, \bar{a}_{t}\left(x, p, p_{t-1}^{s}\right), p_{t-1}^{s}\right)$ in $(x, p)$. Moreover, note that $J_{t}\left(x, p, \bar{a}_{t}\left(x, p, p_{t-1}^{s}\right), p_{t-1}^{s}\right)$ depends on $x$ and $p$ only through $z=x+b p$. Thus, we can define a function $k\left(x+b p, p_{t-1}^{s}\right)=J_{t}\left(x, p, \bar{a}_{t}\left(x, p, p_{t-1}^{s}\right), p_{t-1}^{s}\right)$ in which $b>0$. It then follows by Lemma 1 that the second term of $K_{t}\left(x, p, p_{t-1}^{s}\right)$ is submodular in $(x, p)$. Hence the submodularity of $K_{t}\left(x, p, p_{t-1}^{s}\right)$ follows.

Part (b) follows directly from the submodularity and concavity of $K_{t}\left(x, p, p_{t-1}^{s}\right)$.

For part (c), it is sufficient to show that $x-\theta+$ $b p^{*}(x)$ is increasing in $x$ as $a_{t}^{i}\left(x, p, p_{t-1}^{s}\right)=a_{t}^{i}(x-\theta+$ $\left.b p, p_{t-1}^{s}\right)$ is decreasing in $x$ and $p$. To that end, let $z=x-\theta+b p$ so $J_{t}\left(x, p, \bar{a}_{t}\left(x, p, p_{t-1}^{s}\right), p_{t-1}^{s}\right)$ can be denoted as $J_{t}\left(z, \bar{a}_{t}\left(z, p_{t-1}^{s}\right), p_{t-1}^{s}\right)$. Define

$$
\begin{aligned}
\tilde{K}_{t}\left(z, x, p_{t-1}^{s}\right)= & K_{t}\left(x, p, p_{t-1}^{s}\right)=\frac{z-x+\theta}{b} \mathrm{E}\left[x-z+\varepsilon_{t}\right] \\
& +J_{t}\left(z, \bar{a}_{t}\left(z, p_{t-1}^{s}\right), p_{t-1}^{s}\right),
\end{aligned}
$$

which is supermodular in $x$ and $z$. Therefore, $z^{*}(x)=$ $x-\theta+b p^{*}(x)$ is increasing in $x$. So the desired result follows.

Theorem 5 states that, when the demand function is additive, the optimal price of each period decreases with the starting inventory level. Moreover, there exist multiple possible list prices, each of which corresponds to the selling price when the inventory is replenished to one of the thresholds. These structural results for the optimal policy extend those of Federgruen and Heching (1999) who show that a base-stock list price policy is optimal in the setting without using portfolio procurement and linear replenishment cost.

\section{Multi-Period Option Contract}

In the previous discussion, the firm can adjust its option-reservation quantities from suppliers in each period, and therefore can respond to market demand agilely. However, in some circumstances, suppliers may not be able to offer such flexibility to their cus- tomers, because it may be impossible or too costly for the suppliers to change their supply capacity from period to period. Suppliers may have to use overtime, temporary labor, or transfer capacities reserved for other buyers to cover the fluctuations, which will increase their costs. Hence, the suppliers may prefer the firm to fix a constant number of options for multiple periods and even offer the firm a more competitive price for doing so in order to better schedule their production as well as labor/machines. This motivates us to study the case where the suppliers and the firm sign option contracts in which the reserved options run for a given number of periods, so that suppliers need not change their capacity for a particular buyer too frequently.

For tractability, we assume that the number of periods covered by the option contract from each supplier is the same and denoted by $L$, which we also call reservation cycle. Then at the beginning of periods $1, L+1,2 L+1, \ldots$, which we call option-reservation periods, the firm can decide the option-reservation quantities $\bar{a}_{k L+1}=\left(a_{k L+1}^{1}, \ldots, a_{k L+1}^{N}\right), k=0,1,2, \ldots$ (for simplicity, we denote $\bar{a}_{k L+1}$ by $\bar{a}_{k}$ ). In every period within a reservation cycle $k$, the firm has $\bar{a}_{k}$ options available hence it can exercise up to $a_{k}^{i}-a_{k}^{i-1}$ options from supplier $i$. In other words, the available options in period $t, t=k L+1, k L+2, \ldots,(k+1) L$ are all equal to $\bar{a}_{k}$. In such a setting, the option-reservation prices $C_{t}^{i}$ are defined on the reservation periods, which are nonstationary over periods and may depend on the realization of the spot market price in the previous period. Furthermore, as we require that the reservation quantity of each period be the same in the reservation cycle from each supplier, the total reservation quantity of the firm is $\sum_{i=1}^{N} L\left(a_{k}^{i}-a_{k}^{i-1}\right)=L a_{k}^{N}$ with the total reservation $\operatorname{cost} \sum_{i=1}^{N} C_{k}^{i} L\left(a_{k}^{i}-a_{k}^{i-1}\right)$. It is clear that, when $L=1$, it reduces to our original model.

Let the total length of the planning horizon be an integer multiple of the reservation cycle $L$, i.e., $T=K L$ with $K \in \mathbf{Z}^{+}$. Define $\tilde{V}_{t}\left(x, p_{t-1}^{s}\right)$ as the optimal total profit of the firm from period $t$ to the end of the planning horizon under this multi-period option setting. We formulate the dynamic program as follows. For $k=0,1, \ldots, K-1$,

$$
\begin{aligned}
& \tilde{V}_{k L+1}\left(x, p_{k L}^{s}\right)= \\
& \mathrm{E}_{C_{k}}\left[\max _{\substack{a_{k}^{N} \geq a_{k}^{N-1} \geq \cdots \geq a_{k}^{1} \geq 0 \\
\text { a }}}\left\{-\sum_{i=1}^{N} C_{k}^{i} L\left(a_{k}^{i}-a_{k}^{i-1}\right)+W_{k L+1}\left(x, \bar{a}_{k}, p_{k L}^{s}\right)\right\}\right],
\end{aligned}
$$

where the first term is the reservation cost for the following $L$ periods and

$$
\begin{gathered}
W_{k L+j}\left(x, \bar{a}_{k}, p_{k L+j-1}^{s}\right)=\max _{p \in[p, \bar{p}]}\left\{\mathrm { E } _ { \varepsilon _ { k L + j } , P _ { k L + j } ^ { s } } \left[p D_{k L+j}\left(p, \varepsilon_{k L+j}\right)\right.\right. \\
\left.\left.+U_{k L+j}\left(x-D_{k L+j}\left(p, \varepsilon_{k L+1}\right), \bar{a}_{k}, P_{k L+j}^{s}\right)\right]\right\}
\end{gathered}
$$


for $j=1,2, \ldots, L-1$, is the profit-to-go function, with $U_{k L+j}$ defined by

$$
\begin{aligned}
U_{k L+j}\left(x^{\prime}, \bar{a}_{k}, p_{k L+j}^{s}\right)= & \max _{y \geq x^{\prime}}\left\{-\mathscr{C}_{k L+j}^{E}\left(y-x^{\prime}, \bar{a}_{k}, P_{k L+j}^{s}\right)\right. \\
& \left.-G_{k L+j}(y)+\tilde{W}_{k L+j+1}\left(y, \bar{a}_{k}, p_{k L+j}^{s}\right)\right\},
\end{aligned}
$$

where $\tilde{W}_{k L+j+1}\left(x, \bar{a}_{k}, p_{k L+j}^{s}\right):=W_{k L+j+1}\left(x, \bar{a}_{k}, p_{k L+j}^{s}\right)$ for $j=1, \ldots, L-1$, and $\tilde{W}_{k L+j+1}\left(x, \bar{a}_{k}, p_{k L+j}^{s}\right):=\tilde{V}_{(k+1) L+1}(x$, $\left.p_{(k+1) L}^{s}\right)$ for $j=L$ and $k=0,1, \ldots, K-1$. The terminal condition is $\tilde{W}_{K L+1}\left(x, \bar{a}, p_{K L}^{s}\right) \equiv 0$.

For each period $t$, if it is a reservation period ( $t=k L+1$ for some $0 \leq k \leq K-1$ ), then the firm makes three decisions: the option-reservation quantities from the suppliers for the following $L$ periods, the selling price of the product for the current period, and the inventory replenishment quantity after demand and the spot price are realized. If period $t$ is not a reservation period, only the latter two decisions need to be made based on the reserved options. Note that, for the ease of presentation, we swap the sequence of the pricing and reservation decisions in a reservation period in this setting compared with the original model.

Most of the results for the single-period option contracts problem, reported in sections 4,5 , and 6 , can be extended to the multi-period option contracts scenario. Because of their similarities, we here only briefly discuss the results without presenting their proofs. We can show that $\tilde{V}_{t}\left(x, p_{t-1}^{s}\right), W_{t}\left(x, \bar{a}, p_{t-1}^{s}\right)$, $\tilde{W}_{t}\left(x, \bar{a}, p_{t-1}^{s}\right)$, and $U_{t}\left(x^{\prime}, \bar{a}, p_{t}^{s}\right)$ are all concave functions. Furthermore, because in each period after the demand and the spot market price are realized, the firm still faces a linear piecewise convex ordering cost, the structure of the optimal replenishment policy for each period remains the same as that presented in Theorem $2 .^{3}$ It can also be shown that the optimal price in each period is decreasing in the initial inventory level $x$ when demand function is additive. However, the computation of the optimal reservation quantities will become more complicated since one needs to first optimize the inventory replenishment and pricing policies of $L$ periods for any given $\bar{a}$.

\section{Numerical Study}

In this section, we conduct a numerical study to provide some important insights. We first present comparative statics results of system parameters followed by a discussion on the benefit of portfolio procurement and dynamic pricing. After that, we compare the results of the base model vis-à-vis the one discussed in section 7 with $L=2$. Finally, we develop a simple heuristic for computing near-optimal reservation quantities and test its effectiveness numerically.
We study a system with two suppliers $N=2$ together with a spot market. We assume the cost function for inventory holding and customer backlog is $G(y)=$ $h y^{+}+s y^{-}$, where $h$ is the one-period unit holding cost and $s$ is the one-period unit shortage cost. To reduce the dimension of the problem and for computational simplicity, throughout this numerical study except the second part of section 8.2, we use deterministic but nonstationary reservation prices and assume that the spot market price $P_{t}^{s}$ is random and uniformly distributed over $[13,23]$ (so $E\left[P_{t}^{s}\right]=18$ ). Hence, we can drop the state $p_{t-1}^{s}$ in the value function $V_{t}$ as the firm no longer needs to keep track of it. We consider a base example with parameters $s=30, h=4.4, c_{t}^{1}=6, c_{t}^{2}=2+0.5 t$, $e_{t}^{1}=t+2, \quad e_{t}^{2}=1.5 t+5.2, \quad$ and additive demand $D=(\theta-b p)+\varepsilon$, where $\theta=40, b=2, \varepsilon$ is uniformly distributed with support $[0,30]$. The planning horizon $T=3$.

\subsection{Comparative Statics}

We present computational results that illustrate the sensitivity of the optimal expected profit of the firm and the corresponding optimal reservation quantities, base-stock levels, and selling prices on different system parameters. We focus on the inventory holding cost rate $h$, the option-reservation price $c_{t}^{1}$ of supplier 1 , the exercising price $e_{t}^{2}$ of supplier 2 , and the length of the planning horizon $T$. For $e_{t}^{2}$, we change the value $z$ of $e_{t}^{2}-e_{t}^{1}=0.5 t+z$. Moreover, to see the impact of demand variance, we keep the expected value of $\varepsilon$ at 15 while changing the corresponding lower and upper bounds of its support to generate different levels of variance. We generate different instances by altering the focal parameter while fixing other parameters at those of the base case. The results are tabulated in Tables 1 and 2. Note that, because the backlog cost is higher than the spot price and both of them are stationary, $\tilde{S}_{t}\left(p_{t}^{s}\right)=0$ and so we omit it in the tables. Unless otherwise noted, the optimal reservation quantities $a_{1}^{i *}$, price $p^{*}$, and profit $V_{1}$ reported in the tables are values at $x=10$ as they depend on $x$.

In Table 1, it is clear that the optimal profit of the firm, the optimal reservation quantity $a_{1}^{1 *}$ from supplier 1, and the optimal replenishment thresholds decrease while the reservation quantity $a_{1}^{2 *}-a_{1}^{1 *}$ increases when the holding cost $h$ increases. In addition, when the unit exercising cost $e_{t}^{2}$ of supplier 2 increases, the optimal profit of the firm, the threshold $S_{1}^{2}$, and the optimal reservation quantity $a_{1}^{2 *}-a_{1}^{1 *}$ from supplier 2 decrease while the optimal reservation quantity $a_{1}^{1 *}$ from supplier 1 increases. Although it is not shown in the tabulated results due to the limited space, we observe that the optimal price is nonincreasing in $h$ and nondecreasing in $e_{t}^{2}$ from the numerical results with different state variable $x$.

When the unit reservation price $c_{t}^{1}$ of supplier 1 increases, from Table 2, the optimal profit of the firm 
Table 1 Comparative Statics: Holding Cost $h$ and Exercising Price $e_{t}^{2}$ of Supplier 2

\begin{tabular}{rrrrrrrrrrrrrrr}
\hline$h$ & $S_{1}^{1}$ & $S_{1}^{2}$ & $a_{1}^{1 *}$ & $a_{1}^{2 *}-a_{1}^{1 *}$ & $p_{1}^{*}$ & $V_{1}$ & $z$ & $S_{1}^{1}$ & $S_{1}^{2}$ & $a_{1}^{1 *}$ & $a_{1}^{2 *}-a_{1}^{1 *}$ & $p_{1}^{*}$ & $V_{1}$ \\
\hline 3.2 & 32 & 20 & 18 & 0 & 18 & 436.17 & 2 & 29 & 6 & 0 & 18 & 18 & 449.27 \\
3.6 & 32 & 0 & 13 & 5 & 18 & 430.44 & 2.5 & 29 & 0 & 0 & 18 & 18 & 437.66 \\
4.0 & 30 & 0 & 2 & 16 & 18 & 427.69 & 3 & 29 & 0 & 0 & 18 & 18 & 429.06 \\
4.4 & 29 & 0 & 0 & 18 & 18 & 426.06 & 3.5 & 29 & 0 & 5 & 12 & 18 & 422.47 \\
4.8 & 26 & 0 & 0 & 18 & 18 & 424.76 & 4 & 29 & 0 & 10 & 7 & 18 & 419.14 \\
\hline
\end{tabular}

and the optimal reservation quantity from supplier 1 decrease; the optimal selling price $p_{1}^{*}$, the optimal reservation quantity from supplier 2 , the replenishment thresholds $S_{1}^{1}$ and $S_{1}^{2}$ increase. It is always interesting to see the impact of demand variance. By changing the lower and upper bounds of the support of $\varepsilon$, we get five different levels of variance while keeping $\mathrm{E}[\varepsilon]=15$. We find that the optimal profit decreases while the inventory replenishment thresholds and the total reservation quantity increase as $\varepsilon$ becomes more variable. For the reservation quantity from each individual supplier, the optimal reservation quantity $a_{1}^{1 *}$ from supplier 1 decreases with demand variance while the optimal reservation quantity from supplier 2 increases with demand variance. The selling price is nondecreasing in the variance of $\varepsilon$. The decrease of the optimal profit is expected as the demand becomes more variable, which makes it harder to match supply and demand and thus results in a lower profit. In addition, a higher reservation quantity can mitigate the demand variance while a higher price can reduce the expected demand.

Finally, we run the base example for different lengths of the planning horizon $T$ for $T \in\{3,5,7,9\}$ but change the cost parameters to be stationary, i.e., $c_{t}^{2}=2, e_{t}^{1}=2$, and $e_{t}^{2}=5.2$. We find that the optimal profit, reservation quantities, and threshold levels are increasing in $T$, but these optimal control parameters converge to certain constants when $T$ is longer than 5 .

\subsection{Benefit of Portfolio Procurement and Dynamic Pricing}

In this section, we aim to quantify the benefit of two major features of the model we study. First, how much the firm can gain by adopting portfolio procurement with two suppliers vis-à-vis a system with just one supplier? Second, how much the firm can benefit from dynamic pricing comparing to static pricing?

We start by examining the first issue. By alternating the holding cost rate $h$ from 3.2 to 4.8 with step size 0.4 and $c_{t}^{1}$ from 4 to 8 with step size 1 of the previous base case, we compare the resulting profits between the cases where the firm can procure from two suppliers and where it can only procure from one of the two. Let $V_{1}^{i}(x)$ denote the optimal profit of the firm when solely ordering from supplier $i, i=1,2$ throughout the horizon. We define the benefit of the firm when the starting inventory level is $x, x \in[-9,40]$ as

$$
B_{0}(x)=\frac{V_{1}(x)-\max \left\{V_{1}^{1}(x), V_{1}^{2}(x)\right\}}{V_{1}(x)} \times 100 \% .
$$

In general, we find that the benefit $B_{o}(x)$ decreases with the starting inventory level $x$, which is intuitive as more inventory means less need to order and so less valuable of the flexibility from dual sourcing. The largest benefit among the instances (among all the combinations of different system parameters and different initial state $\mathrm{x}$ ) we tested is $6.46 \%$.

We plot the average benefit $\bar{B}_{0}$ over inventory level $[-9,40]$, i.e., $\bar{B}_{0}=\sum_{x=-9}^{40} B_{0}(x) / 50$ to show the trend of the benefit with respect to $h$ and $c_{t}^{1}$ respectively. In Figure 1 (a), we can observe that the benefit of portfolio procurement increases as the holding cost $h$ increases. This implies that, when the holding cost of inventory is high, the firm can benefit more by keeping a larger portfolio (supply base) to have a higher flexibility. Moreover, with the increase of the reservation price $c_{t}^{1}$ of supplier 1 , the average benefit of portfolio procurement $\bar{B}_{0}$ first increases then decreases. This can be explained as follows. When $c_{t}^{1}$ increases but is still of moderate value, if the firm can only source from one supplier, it will pick supplier 1 .

Table 2 Comparative Statics: Reservation Price $c_{t}^{1}$ of Supplier 1 and Demand Variance $\operatorname{Var}(\varepsilon)$

\begin{tabular}{rrrrrrrrrrrrrrr}
\hline$c_{t}^{1}$ & $S_{1}^{1}$ & $S_{1}^{2}$ & $a_{1}^{1 *}$ & $a_{1}^{2 *}-a_{1}^{1 *}$ & $p_{1}^{*}$ & $V_{1}$ & $\operatorname{Var}(\varepsilon)$ & $S_{1}^{1}$ & $S_{1}^{2}$ & $a_{1}^{1 *}$ & $a_{1}^{2 *}-a_{1}^{1 *}$ & $p_{1}^{*}$ & $V_{1}$ \\
\hline 4 & 25 & 0 & 17 & 3 & 17 & 515.32 & $4(12,18)$ & 24 & 0 & 7 & 4 & 18 & 523.46 \\
5 & 28 & 0 & 11 & 7 & 18 & 463.75 & $10(10,20)$ & 26 & 0 & 5 & 7 & 18 & 508.05 \\
6 & 29 & 0 & 0 & 18 & 18 & 426.06 & $24(7,23)$ & 28 & 0 & 4 & 10 & 18 & 484.62 \\
7 & 30 & 0 & 0 & 18 & 18 & 405.02 & $36.67(5,25)$ & 28 & 0 & 2 & 13 & 18 & 468.37 \\
8 & 30 & 6 & 0 & 18 & 18 & 400.51 & $60.67(2,28)$ & 29 & 0 & 0 & 17 & 18 & 443.19 \\
\hline
\end{tabular}


Figure 1 Value of (a) Portfolio Procurement and (b) Dynamic Pricing: Deterministic $C_{t}$

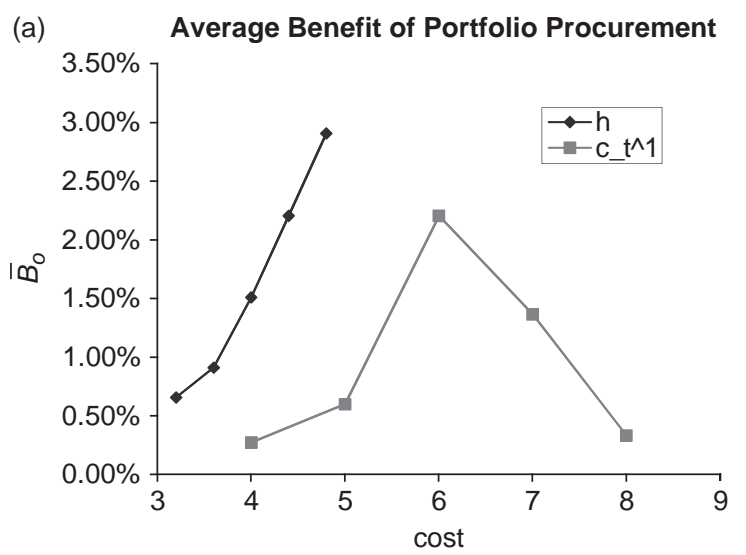

But the firm with two suppliers would reserve from both suppliers to enjoy the flexibility of supplier 2. So the firm gains more benefit when $c_{t}^{1}$ increases. Nevertheless, after $c_{t}^{1}$ becomes sufficiently large, the firm with a single supplier will choose supplier 2 while the firm with two suppliers will reserve most of the quantity from supplier 2 as well. This leads to a decrease in the benefit of portfolio procurement. Furthermore, we find that the benefit of portfolio procurement increases with demand variance. The average benefit $\bar{B}_{0}$ to the firm when $\operatorname{Var}(\varepsilon)$ changes from 4 to 60.67 (as those in Table 2) is, respectively, $0.34 \%, 0.57 \%, 0.86 \%, 1.26 \%$, and $2.27 \%$.

We now investigate how much the firm can gain by adopting dynamic pricing in lieu of static pricing strategy. Let $V_{1}^{s p}(x)$ be the optimal profit of the firm by using the optimal static price throughout the horizon when the starting inventory level is $x$. Analogously, we define the benefit of dynamic pricing $B_{p}(x)$ as

$$
B_{p}(x)=\frac{V_{1}(x)-V_{1}^{s p}(x)}{V_{1}(x)} \times 100 \% .
$$

(b)

Average Benefit of Dynamic Pricing

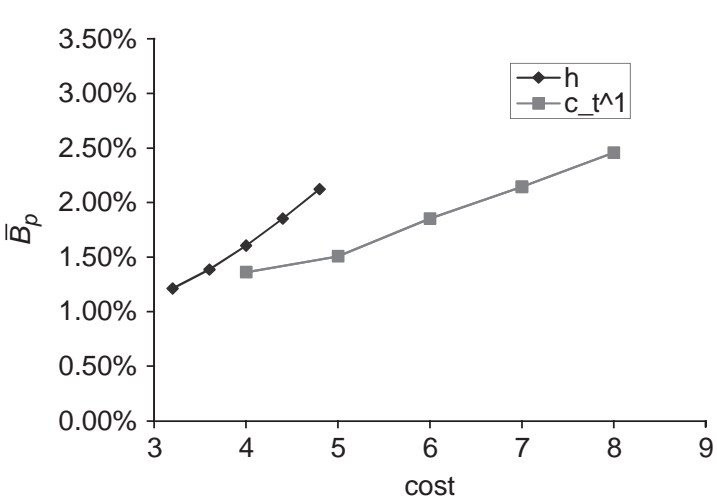

From the numerical examples tested, we find that the value of dynamic pricing is usually lower when the inventory level $x$ is intermediate than when the inventory level is low or high. This is because the optimal dynamic pricing strategy tends to keep a stable price when the inventory level is intermediate. By also alternating the value of holding cost $h$ or reservation price $c_{t}^{1}$ as in the previous study, we plot the average value of dynamic pricing $\bar{B}_{p}$, i.e., $\bar{B}_{p}=\sum_{x=11}^{70}$ $B_{p}(x) / 60$ in Figure $1 b$.

The figure illustrates that the value of dynamic pricing is increasing in the holding cost $h$ and the reservation price $c_{t}^{1}$ of supplier 1 . That is, the higher the inventory holding or reservation costs, the more valuable it is for the firm to dynamically adjust selling price to balance supply and demand. To see the change of $\bar{B}_{p}$ when the variance of demand increases, we further calculate the expected profit of the firm under optimal static pricing with five different variances of $\varepsilon$. We find that the firm will benefit slightly more from dynamic pricing when demand is more variable. Specifically, the average benefit $\bar{B}_{p}$ for each value of $\operatorname{Var}(\varepsilon)$ from 4 to 60.67 is $1.65 \%, 1.65 \%, 1.67 \%$,

Figure 2 Value of (a) Portfolio Procurement and (b) Dynamic Pricing: Random and Spot-Dependent $c_{t}$
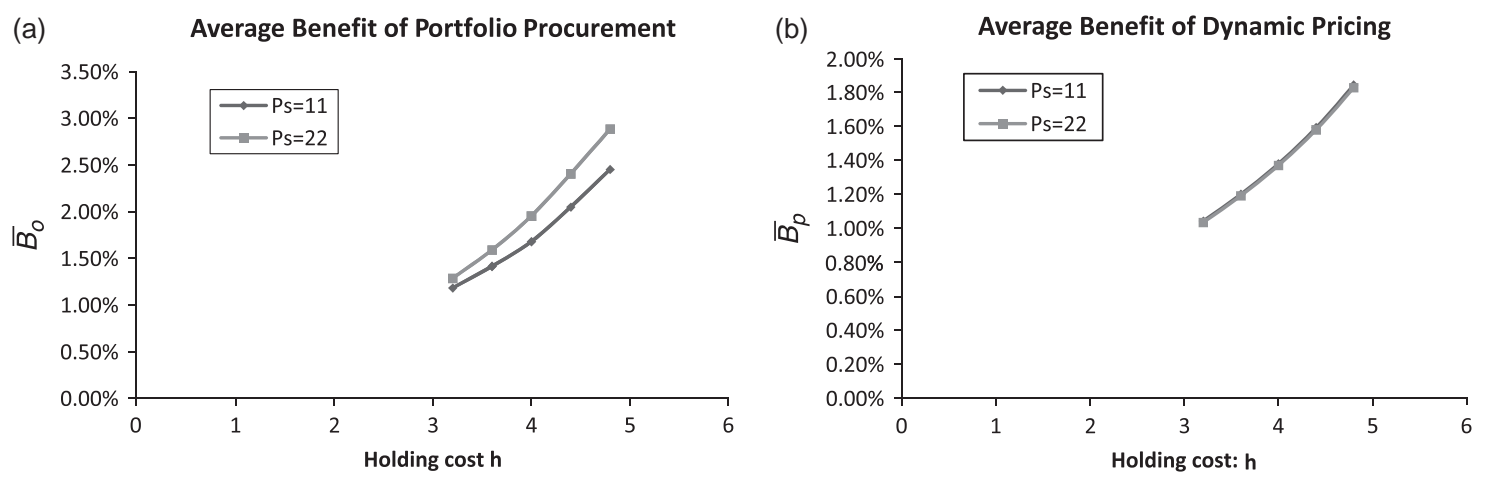
Figure 3 Value of (a) Portfolio Procurement and (b) Dynamic Pricing: Random and Spot-Dependent $C_{t}$

(a)

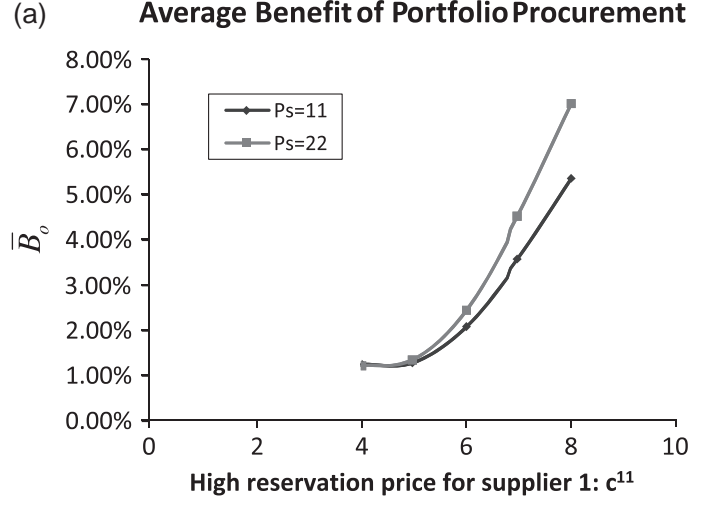

(b)

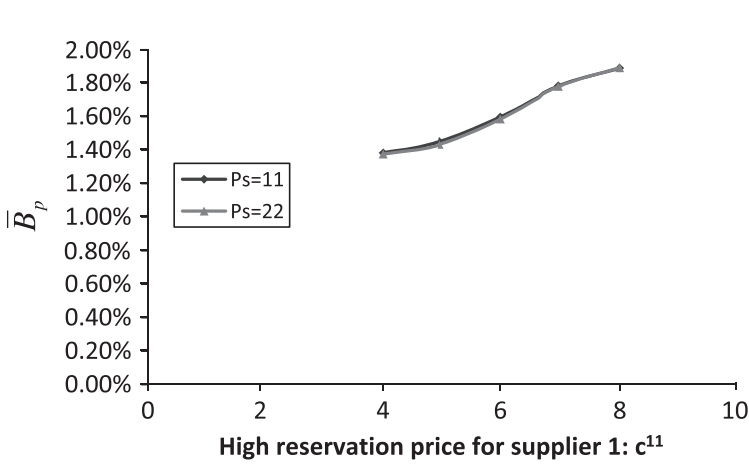

$1.69 \%$, and $1.78 \%$. The intuition is clear, the more volatile the market environment, the more important it becomes for the firm to dynamically adjust its selling price. Moreover, compared with the benefit of portfolio procurement, the value of dynamic pricing is less sensitive to the demand variability.

So far in the numerical study we have ignored the dependence between the reservation prices $C_{t}$ and the spot price $P_{t}^{s}$. To obtain more insights on the benefits of dynamic pricing and portfolio procurement, we design additional numerical examples that take into account such dependence. Assume the spot price $\left\{P_{t}^{s}\right.$, $1 \leq t \leq T\}$ forms a two-state discrete Markov chain $P_{t}^{s} \in\left\{p_{s 1}, p_{s 2}\right\}$ with $p_{s 1}=11$ and $p_{s 2}=22$. The transition probabilities are given as $p_{11}=0.3, p_{12}=0.7, p_{21}=0.4$, and $p_{22}=0.6$. For example, when the previous period's spot price is $p_{s 1}$, the probability that it is still $p_{s 1}$ in the current period is $p_{11}=0.3$. As a result, the steady state probabilities are $p_{1}=4 / 11$ and $p_{2}=7 / 11$ and the expected spot price $\mathbf{E}\left[P_{t}^{s}\right]=18$. We also assume two possible reservation prices $\left(c^{11}, c^{12}\right)=(6$, 3 ) and $\left(c^{21}, c^{22}\right)=(4,2)$. When $P_{t-1}^{s}=p_{s 1}$, with probability 0.2, $C_{t}^{i}=c^{1 i}$ and with probability $0.8, C_{i}^{t}=c^{2 i}$; when $P_{t-1}^{s}=p_{s 2}$, with probability $0.6, C_{i}^{t}=c^{1 i}$ and with probability $0.4, C_{i}^{t}=c^{2 i}$. This follows from that a higher (lower) spot price in the previous period is more likely to lead to a higher (lower) reservation price in the current period. The other system parameters are the same as the previous basic setting.
We first examine the benefit of portfolio procurement and dynamic pricing by alternating the holding cost $h$ and the results are reported in Figure 2. In each sub-figure, there are two curves, each of which represents the scenario with a different starting spot market price in period one. As can be seen from Figure 2a, the average benefit of portfolio procurement is $1-3 \%$ and still increases in the holding cost $h$. Figure $2 \mathrm{~b}$ demonstrates that the benefit of dynamic pricing also increases with the holding cost but is rather insensitive to the different starting spot prices.

We then investigate the impact of the spread of high and low reservation prices, by increasing $c^{11}$ from 4 to 8 with step size one. The results are illustrated in Figure 3. Figure 3a shows that the benefit of portfolio procurement is quite sensitive to the price spread and increases from $1.21 \%$ to $7.01 \%$ when the starting spot price is 22. However, the dynamic pricing still brings about $1-2 \%$ of the benefit to the firm and the benefit is not very sensitive to the price spread of the high and low reservation prices.

\subsection{Two-Period Options}

The analytical results in section 7 show that the structure of the optimal policy will not change when suppliers offer options that are fixed for $L>1$ periods. We intend to see how the optimal policy parameters differ from the case $L=1$ and how much it affects the

Table 3 Comparison of Option Contracts: $L=1$ vs. $L=2$

\begin{tabular}{lcccccccccccccc}
\hline$h$ & $a_{1}^{1 *}$ & $a_{1}^{2 *}-a_{1}^{1 *}$ & $p_{1}^{*}$ & $\tilde{V}_{1}$ & $V_{1}$ & Gain\% & $c_{t}^{1}$ & $a_{1}^{1 *}$ & $a_{1}^{2 *}-a_{1}^{1 *}$ & $p_{1}^{*}$ & $\tilde{V}_{1}$ & $V_{1}$ & Gain\% \\
\hline 3.2 & 4 & 15 & 18 & 509.58 & 531.81 & 4.36 & 4 & 18 & 3 & 17 & 620.98 & 631.05 & 1.62 \\
3.6 & 4 & 15 & 18 & 506.40 & 521.93 & 3.07 & 5 & 11 & 10 & 17 & 550.86 & 564.46 & 2.47 \\
4.0 & 3 & 17 & 18 & 503.72 & 515.62 & 1.77 & 6 & 3 & 17 & 18 & 501.33 & 511.73 & 2.07 \\
4.4 & 3 & 17 & 18 & 501.33 & 511.73 & 2.07 & 7 & 0 & 20 & 18 & 476.01 & 480.90 & 1.03 \\
4.8 & 3 & 17 & 18 & 499.42 & 508.97 & 1.91 & 8 & 0 & 20 & 18 & 463.56 & $468, .34$ & 1.03 \\
\hline
\end{tabular}


firm's profit when imposing options contracts to cover more than one period. Clearly, the profit value of the firm will decrease in $L$. In the following numerical study, we consider the case with two suppliers and the options are fixed for two consecutive periods, i.e., $L=2$.

Define the percentage gain of the firm by switching from $L=2$ to $L=1$ as

$$
\left(V_{1}(x)-\tilde{V}_{1}(x)\right) / V_{1}(x) \times 100 \%
$$

and recall that $V_{1}(x)$ is the optimal profit over the planning horizon for $L=1$ while $\tilde{V}_{1}(x)$ is the optimal profit over the planning horizon for $L=2$, both starting from the initial inventory level $x$. The same set of parameters as those in the previous section are used but with $T=4$. We mainly conduct comparisons with different values of $h$ and $c_{t}^{1}$. Note that, to have a fair comparison, when $L=1$, we set $c_{2}^{1}=c_{1}^{1}$ and $c_{4}^{1}=c_{3}^{1}$ for the system. As $T=4$, the firm with $L=2$ only has two opportunities to reserve from suppliers. The results are reported in Table 3.

The tabulated results are for $x=10$. Among all the instances tested with different combinations of system parameters and the initial state $x \in[1,70]$, the average percentage gain of the more flexible option contracts $(L=1)$ over the less flexible ones $(L=2)$ is $4.76 \%$ with the largest gain at $10.05 \%$. The dependence of the percentage gain on system parameters seems to have no clear pattern. Furthermore, we observe that the firm tends to reserve more and set a lower selling price when $L=2$ than when $L=1$. For example, when $c_{t}^{1}=4$ and $x=10$, the firm reserves $a_{1}^{1 *}=18$ and $a_{1}^{2 *}=$ 21 (three units from supplier 2) when $L=2$ while it reserves $a_{1}^{1 *}=17$ and $a_{1}^{2 *}=20$ when $L=1$; the selling prices $p_{1}^{*}$ for $L=2$ and $L=1$ are 17 and 18 , respectively.

\subsection{Heuristic for Reservation Quantity}

Because the computation of the optimal reservation quantity $\bar{a}_{t}\left(x, p, p_{t-1}^{s}\right)$ is rather complicated, in this section, we provide a simple heuristic for computing near-optimal solutions and test its effectiveness numerically. For the additive demand, with $z=$ $x+b p-\theta$, let $\tilde{a}_{t}^{0}\left(z, p_{t-1}^{s}\right)=0$ and $\tilde{a}_{t}^{i}(z), i=1, \ldots, N-1$, be the solution of the following equation.

$$
\begin{aligned}
\tilde{a}_{t}^{i}\left(z, p_{t-1}^{s}\right)= & \min \left\{a^{i} \geq \tilde{a}_{t}^{i-1}(z): c_{t}^{i}-c_{t}^{i+1} \geq \mathrm{E}_{P_{t}^{s}}\right. \\
& {\left[\mathbf { 1 } ( P _ { t } ^ { s } \geq e _ { t } ^ { i + 1 } ) \left(\int_{z-S_{t}^{i}\left(P_{t}^{s}\right)+a^{i}}^{z-S_{t}^{i+1}\left(P_{t}^{s}\right)+a^{i}}-G_{t}^{\prime}\left(z-\xi+a^{i}\right) f(\xi) d \xi\right.\right.} \\
& \left.\left.+\left(e_{t}^{i+1}-e_{t}^{i}\right) \bar{F}\left(z-S_{t}^{i+1}\left(P_{t}^{s}\right)+a^{i}\right)\right)\right] \\
& +\mathrm{E}_{P_{t}^{s}}\left[\mathbf { 1 } ( e _ { t } ^ { i } \leq P _ { t } ^ { s } < e _ { t } ^ { i + 1 } ) \left(\int_{z-S_{t}^{i}\left(P_{t}^{s}\right)+a^{i}}^{z-\tilde{s}_{t}\left(P_{t}^{s}\right)+a^{i}}-G_{t}^{\prime}\left(z-\xi+a^{i}\right)\right.\right. \\
& \left.\left.\left.f(\xi) d \xi+\left(P_{t}^{s}-e_{t}^{i}\right) \bar{F}\left(z-\tilde{S}_{t}\left(P_{t}^{s}\right)+a^{i}\right)\right)\right]\right\}
\end{aligned}
$$

and for $\tilde{a}_{t}^{N}\left(z, p_{t-1}^{s}\right)$, it satisfies

$$
\begin{aligned}
\tilde{a}_{t}^{N}\left(z, p_{t-1}^{s}\right)= & \min \left\{a^{N} \geq \tilde{a}_{t}^{N-1}(z): c_{t}^{N} \geq \mathrm{E}_{P_{t}^{s}}\right. \\
& {\left[\mathbf { 1 } ( e _ { t } ^ { N } \leq P _ { t } ^ { s } ) \left(\int_{z-S_{t}^{N}\left(P_{t}^{s}\right)+a^{N}}^{z-\tilde{S}_{(}\left(P_{t}^{s}\right)+a^{N}}-G_{t}^{\prime}\left(z-\xi+a^{N}\right) f(\xi) d \xi\right.\right.} \\
& \left.\left.\left.+\left(P_{t}^{s}-e_{t}^{N}\right) \bar{F}\left(z-\tilde{S}_{t}\left(P_{t}^{s}\right)+a^{N}\right)\right)\right]\right\} .
\end{aligned}
$$

Note that $\tilde{a}_{t}^{i}\left(z, p_{t-1}^{s}\right)$ is easy to solve since each inequality only depends on one decision variable and the right-hand side of the inequality is monotonically decreasing in $a^{i}$. More importantly, the computation does not depend on the future value function $V_{t+1}\left(x, p_{t}^{s}\right)$. To obtain these inequalities, we approximate $\left(V_{t+1}\left(x, p_{t}^{s}\right)\right)^{\prime}$ in $R_{t}^{\prime}\left(x, p_{t}^{s}\right)$ in Equations (3) and (4) by $e_{t}^{i}$ and $e_{t}^{N}$, respectively. The rationale behind is that, in the integrand of each integration in Equation (3), $S_{t}^{i+1}\left(p_{t}^{s}\right) \leq z-\xi+$ $a^{i} \leq S_{t}^{i}\left(p_{t}^{s}\right)$ or $\tilde{S}_{t}\left(p_{t}^{s}\right) \leq z-\xi+a^{i} \leq S_{t}^{i}\left(p_{t}^{s}\right)$. If we assume $S_{t}^{i}\left(p_{t}^{s}\right)$ of different periods $t$ are roughly the same, then for $x$ in the above ranges, it can be shown that $\left(V_{t+1}\right.$ $\left.\left(x, p_{t}^{s}\right)\right)^{\prime} \geq e_{t}^{i}$ from the optimal replenishment policy presented in Theorem 2 and so we simply use $e_{t}^{i}$ to approximate this derivative when solving $\tilde{a}_{t}^{i}\left(z, p_{t-1}^{s}\right)$. We can similarly derive the heuristic reservation quantities when demand is multiplicative. After obtaining $\tilde{a}_{t}^{i}\left(z, p_{t-1}^{s}\right)$, we plug them into the original recursion

Table 4 Performance of the Heuristic

\begin{tabular}{lcccccccc}
\hline$h$ & $E_{h}(\%)$ & $E_{h}^{m}(\%)$ & $c_{t}^{1}$ & $E_{h}(\%)$ & $E_{h}^{m}(\%)$ & $\operatorname{Var}(\varepsilon)$ & $E_{h}(\%)$ & 0.21 \\
\hline 3.2 & 0.68 & 1.40 & 4 & 0.70 & 1.78 & 4 & 0.67 \\
3.6 & 0.53 & 0.98 & 5 & 1.53 & 2.64 & 10 & 0.22 \\
4 & 0.33 & 0.66 & 6 & 0.25 & 0.60 & 24 & 0.26 \\
4.4 & 0.25 & 0.60 & 7 & 0.02 & 0.03 & 36.67 & 0.41 \\
4.8 & 0.16 & 0.50 & 8 & 0.26 & 0.60 & 60.67 & 0.55 \\
\hline
\end{tabular}


$K_{t}\left(x, p, p_{t-1}^{s}\right)$ and further compute the corresponding optimal selling price. This procedure leads to a set of heuristic solutions for all the decisions.

We evaluate the effectiveness of the heuristic over the instances we tested in section 8.1. We consider the starting inventory level $x$ ranging from -9 to 40 and let the average and maximum relative error, $E_{h}$ and $E_{h}^{m}$, be defined, respectively, as

$$
\begin{aligned}
& E_{h}=\frac{1}{50} \sum_{x=-9}^{40}\left[\frac{V_{1}(x)-V_{1}^{h}(x)}{V_{1}(x)}\right] \times 100 \%, \\
& E_{h}^{m}=\max _{x \in[-9,40]}\left\{\frac{V_{1}(x)-V_{1}^{h}(x)}{V_{1}(x)} \times 100 \%\right\},
\end{aligned}
$$

where $V_{1}^{h}(x)$ is the resulting profit of the heuristic policy. Under these measures, among all the instances we tested, the average relative error is $0.42 \%$ while the largest relative error is $2.64 \%$. We can see that the overall performance of the heuristic is quite robust to the system parameters. When the variance of demand increases, the relative error of the heuristic becomes slightly larger. We summarize these numerical results in Table 4. Note that, compared with the results for the case in which only the best supplier is used (Figure 1a), the heuristic policy for the case with two suppliers leads to higher profit for the firm except when $c_{t}^{1}=4$ and $c_{t}^{1}=5$.

\section{Conclusion}

This paper studies combined pricing and portfolio procurement strategies for a multi-period inventory system. A firm procures a single product from a portfolio of supply sources, including a set of option contracts (suppliers) at various levels of flexibility and costs as well as the spot market with uncertain price. Customer demand is random and price sensitive. With the objective of maximizing the total expected profit over a finite planning horizon, the firm makes three decisions in each period, namely, product selling price, option-reservation quantities, and inventory replenishment through exercising the options reserved from the suppliers and ordering from the spot market if needed.

The optimal inventory replenishment policy is shown to be an order-up-to type policy specified by a sequence of thresholds. For the optimal reservation quantities, we analyze some structural properties and show that the cumulative reservation quantity is decreasing in both the starting inventory level and the selling price. A set of optimality conditions are presented for computing the option-reservation quantities. We show that the optimal price is decreasing in the starting inventory level when demand is additive. We also extend the model to the case with multi-period option contracts. Finally, we conduct an extensive numerical study to reveal additional insights of our results. Among others, we quantify the benefit of portfolio procurement and dy- namic pricing and show that both increase with demand variance. Moreover, the value of dynamic pricing is less sensitive to the demand variability. When the reservation prices depend on the realized spot price in the previous period, the value of portfolio procurement increases when the price spread between the high and low reservation prices increases while the value of dynamic pricing is less sensitive to such price spread. A simple heuristic is developed to calculate the nearoptimal reservation quantities and a numerical test shows that the heuristic is effective.

\section{Acknowledgments}

The authors thank the Senior Editor and two anonymous referees for their constructive suggestions that have helped to improve the paper significantly. The first and the fourth authors are supported in part by Hong Kong RGC Earmark Grant 615607 . The second author is supported in part by Hong Kong GRF Grant CUHK 419408. The third author is supported in part by the NSF under CMMI-0800004 and CMMI-0927631.

\section{Appendix. Proofs}

\section{Proof of Theorem 4.}

For period $t$, the optimal $a^{i}$ is the solution of the following optimization problem. Let $a^{0}=0$.

$$
\begin{aligned}
& \max _{\bar{a}} J_{t}\left(x, p, \bar{a}, p_{t-1}^{s}\right) \\
& \text { s.t. } \quad a^{i} \geq a^{i-1}, \quad 1 \leq i \leq N .
\end{aligned}
$$

As $J_{t}\left(x, p, \bar{a}, p_{t-1}^{s}\right)$ is concave and the constraint set is convex, we can apply the Karush-Kuhn-Tucker (KKT) condition (Lundberg 1984) to find the optimal solutions. Let $\bar{\lambda}=\left(\lambda_{1}, \ldots, \lambda_{N}\right)$ and

$$
\begin{aligned}
L\left(x, p, \bar{a}, \bar{\lambda}, p_{t-1}^{s}\right)= & J_{t}\left(x, p, \bar{a}, p_{t-1}^{s}\right)+\lambda_{1} a^{1} \\
& +\lambda_{2}\left(a^{2}-a^{1}\right)+\cdots+\lambda_{N}\left(a^{N}-a^{N-1}\right) .
\end{aligned}
$$

Then, by the KKT condition, the optimal $a^{i}$ should satisfy $\left(L\left(x, p, \bar{a}, \bar{\lambda}, p_{t-1}^{s}\right)\right)_{a^{i}}^{\prime}=0, \quad \lambda_{i}\left(a^{i}-a^{i-1}\right)=0, \quad$ and $\lambda_{i} \geq 0$ for all $i$. Let $\mathbf{1}(A)=1$ if $A$ is true; otherwise, $\mathbf{1}(A)=0$. Based on the possible realization of $P_{t}^{s}$, there are several different cases. We first consider the case $P_{t}^{s}=p_{t}^{s} \geq e_{t}^{i+1}$. For notational convenience, we suppress the $p_{t}^{s}$ in $S_{t}^{i}\left(p_{t}^{s}\right)$ in the following derivation. Note that $x^{\prime}=x-D_{t}\left(p, \varepsilon_{t}\right)$, which is random through $\varepsilon_{t}$. For 


$$
\begin{aligned}
i= & 1, \ldots, N-1, \\
( & \left.\left(x, p, \bar{a}, \bar{\lambda}, p_{t-1}^{s}\right)\right)_{a^{i}}^{\prime}=c_{t}^{i+1}-c_{t}^{i}+\left(\mathrm{E}_{\varepsilon_{t}}\left[U_{t}\left(x^{\prime}, \bar{a}, p_{t}^{s}\right)\right]\right)_{a^{i}}^{\prime} \\
& =c_{t}^{i+1}-c_{t}^{i}+\mathrm{E}_{\varepsilon_{t}}\left[\left[\left(-e_{t}^{i}\left(S_{t}^{i}-x^{\prime}\right)\right.\right.\right. \\
& \left.+\sum_{j=0}^{i-1}\left(e_{t}^{j+1}-e_{t}^{j}\right) a^{j}-G_{t}\left(S_{t}^{i}\right)+V_{t+1}\left(S_{t}^{i}, p_{t}^{s}\right)\right) \\
& \left.\times \mathbf{1}\left(S_{t}^{i}-a^{i-1} \geq x^{\prime}>S_{t}^{i}-a^{i}\right)\right]_{a^{i}}^{\prime} \\
& +\left[\left(-e_{t}^{i} a^{i}+\sum_{j=0}^{i-1}\left(e_{t}^{j+1}-e_{t}^{j}\right) a^{j}-G_{t}\left(x^{\prime}+a^{i}\right)\right.\right. \\
& \left.\left.+V_{t+1}\left(x^{\prime}+a^{i}, p_{t}^{s}\right)\right) \mathbf{1}\left(S_{t}^{i}-a^{i} \geq x^{\prime} \geq S_{t}^{i+1}-a^{i}\right)\right]_{a^{i}}^{\prime} \\
& +\left[\left(-e_{t}^{i+1}\left(S_{t}^{i+1}-x^{\prime}\right)+\sum_{j=0}^{i}\left(e_{t}^{j+1}-e_{t}^{j}\right) a^{j}-G_{t}\left(S_{t}^{i+1}\right)\right.\right. \\
& \left.\left.\left.+V_{t+1}\left(S_{t}^{i+1}, p_{t}^{s}\right)\right) \mathbf{1}\left(S_{t}^{i+1}-a^{i} \geq x^{\prime} \geq S_{t}^{i+1}-a^{i+1}\right)\right]_{a^{i}}^{\prime}\right] \\
& +\mathbf{1}\left(e_{t}^{i+1}-e_{t}^{i}\right) \operatorname{Pr}\left(x^{\prime}<S_{t}^{i+1}-a^{i+1}\right)+\lambda_{i}-\lambda_{i+1} \\
& =\lambda_{i}-\lambda_{i+1}+c_{t}^{i+1}-c_{t}^{i}+\left(e_{t}^{i+1}-e_{t}^{i}\right) P r\left(x^{\prime}<S_{t}^{i+1}-a^{i+1}\right) \\
& +\mathrm{E}_{\varepsilon_{t}}\left[\mathbf { 1 } ( p _ { t } ^ { s } \geq e _ { t } ^ { i + 1 } ) \left(-e_{t}^{i}-G_{t}^{\prime}\left(x^{\prime}+a^{i}\right)\right.\right. \\
& \left.\left.+V_{t+1}^{\prime}\left(x^{\prime}+a^{i}, p_{t}^{s}\right)\right) \mathbf{1}\left(S_{t}^{i}-a^{i} \geq x^{\prime} \geq S_{t}^{i+1}-a^{i}\right)\right],
\end{aligned}
$$

where the last equality can be verified by writing the terms in the form of integration and applying the Lebniz's rule of derivative. For $e_{t}^{i} \leq P_{t}^{s}=p_{t}^{s}<e_{t}^{i+1}$, then

$$
\begin{aligned}
& \left(L\left(x, p, \bar{a}, \bar{\lambda}, p_{t-1}^{s}\right)\right)_{a^{i}}^{\prime}=c_{t}^{i+1}-c_{t}^{i}+\mathrm{E}_{\varepsilon_{t}}\left[\left[-e_{t}^{i}\left(S_{t}^{i}-x^{\prime}\right)+\sum_{j=0}^{i-1}\left(e_{t}^{j+1}-e_{t}^{j}\right) a^{j}\right.\right. \\
& \left.\left.-G_{t}\left(S_{t}^{i}\right)+V_{t+1}\left(S_{t}^{i}, p_{t}^{s}\right)\right] \mathbf{1}\left(S_{t}^{i}-a^{i-1} \geq x^{\prime}>S_{t}^{i}-a^{i}\right)\right]^{\prime} \\
& +\left[\left(-e_{t}^{i} a^{i}+\sum_{j=0}^{i-1}\left(e_{t}^{j+1}-e_{t}^{j}\right) a^{j}-G_{t}\left(x^{\prime}+a^{i}\right)\right.\right. \\
& \left.\left.+V_{t+1}\left(x^{\prime}+a^{i}, p_{t}^{s}\right)\right) \mathbf{1}\left(S_{t}^{i}-a^{i} \geq x^{\prime} \geq \tilde{S}_{t}\left(p_{t}^{s}\right)-a^{i}\right)\right]^{\prime} \\
& +\left[-p_{t}^{s}\left(\tilde{S}_{t}\left(p_{t}^{s}\right)-x^{\prime}\right)+\sum_{j=0}^{i-1}\left(e_{t}^{j+1}-e_{t}^{j}\right) a^{j}+\left(p_{t}^{s}-e_{t}^{i}\right) a^{i}\right. \\
& \left.\left.\left.-G_{t}\left(\tilde{S}_{t}\left(p_{t}^{s}\right)\right)+V_{t+1}\left(\tilde{S}_{t}\left(p_{t}^{s}\right), p_{t}^{s}\right)\right] \mathbf{1}\left(\tilde{S}_{t}\left(p_{t}^{s}\right)-a^{i} \geq x^{\prime}\right)\right]^{\prime}\right]+\lambda_{i}-\lambda_{i+1} \\
& =\lambda_{i}-\lambda_{i+1}+c_{t}^{i+1}-c_{t}^{i}+\mathbf{E}_{\varepsilon_{t}}\left[\left(-e_{t}^{i}-G_{t}^{\prime}\left(x^{\prime}+a^{i}\right)\right.\right. \\
& \left.\left.+V_{t+1}^{\prime}\left(x^{\prime}+a^{i}, p_{t}^{s}\right)\right) \mathbf{1}\left(S_{t}^{i}-a^{i} \geq x^{\prime} \geq \tilde{S}_{t}\left(p_{t}^{s}\right)-a^{i}\right)\right] \\
& +\left(p_{t}^{s}-e_{t}^{i}\right) \operatorname{Pr}\left(\tilde{S}_{t}\left(p_{t}^{s}\right)-a^{i} \geq x^{\prime}\right),
\end{aligned}
$$

where the last equality follows the analogous argument of the previous case. For other realization of $p_{t}^{s},\left(L\left(x, p, \bar{a}, p_{t-1}^{s}\right)\right)_{a^{i}}^{\prime}=c_{t}^{i+1}-c_{t}^{i}+\lambda_{i}-\lambda_{i+1}$. Therefore, combine these cases,

$$
\begin{aligned}
& \left(L\left(x, p, \bar{a}, \bar{\lambda}, p_{t-1}^{s}\right)\right)_{a^{i}}^{\prime}=\lambda_{i}-\lambda_{i+1}+c_{t}^{i+1}-c_{t}^{i}+\left(e_{t}^{i+1}-e_{t}^{i}\right) \mathrm{E}_{P_{t}^{s}} \\
& \quad \times\left[\mathbf{1}\left(P_{t}^{s} \geq e_{t}^{i+1}\right) \operatorname{Pr}\left(x^{\prime}<S_{t}^{i+1}-a^{i+1}\right)\right] \\
& \quad+\mathrm{E}_{\varepsilon_{t}, P_{t}^{s}}\left[\mathbf{1}\left(P_{t}^{s} \geq e_{t}^{i+1}\right)\left(-e_{t}^{i}-G_{t}^{\prime}\left(x^{\prime}+a^{i}\right)+V_{t+1}^{\prime}\left(x^{\prime}+a^{i}, P_{t}^{s}\right)\right)\right. \\
& \left.\quad \times \mathbf{1}\left(S_{t}^{i}-a^{i} \geq x-D_{t}\left(p, \varepsilon_{t}\right) \geq S_{t}^{i+1}-a^{i}\right)\right], \\
& \quad+\mathrm{E}_{\varepsilon_{t}, P_{t}^{s}}\left[\left[\left(-e_{t}^{i}-G_{t}^{\prime}\left(x^{\prime}+a^{i}\right)+V_{t+1}^{\prime}\left(x^{\prime}+a^{i}, P_{t}^{s}\right)\right)\right.\right. \\
& \quad \times \mathbf{1}\left(S_{t}^{i}-a^{i} \geq x^{\prime} \geq \mathbf{1} \tilde{S}_{t}\left(P_{t}^{s}\right)-a^{i}\right)+\left(P_{t}^{s}-e_{t}^{i}\right) \mathbf{1}\left(\tilde{S}_{t}\left(P_{t}^{s}\right)\right. \\
& \left.\left.\quad-a^{i} \geq x^{\prime}\right] \mathbf{1}\left(e_{t}^{i} \leq P_{t}^{s}<e_{t}^{i+1}\right)\right] .
\end{aligned}
$$

Similarly, for $i=N$,

$$
\begin{aligned}
& \left(L\left(x, p, \bar{a}, \bar{\lambda}, p_{t-1}^{s}\right)\right)_{a^{N}}^{\prime}=-c_{t}^{N}+\mathrm{E}_{\varepsilon_{t}, P_{t}^{s}}\left[\left[\left(-e_{t}^{N}-G_{t}^{\prime}\left(x^{\prime}+a^{N}\right)\right.\right.\right. \\
& \left.+V_{t+1}^{\prime}\left(x^{\prime}+a^{N}, P_{t}^{s}\right)\right) \mathbf{1}\left(S_{t}^{N}-a^{N} \geq x^{\prime} \geq \tilde{S}_{t}\left(P_{t}^{s}\right)-a^{N}\right) \\
& \left.\left.+\left(P_{t}^{s}-e_{t}^{N}\right) \mathbf{1}\left(\tilde{S}_{t}\left(P_{t}^{s}\right)-a^{N} \geq x^{\prime}\right)\right] \mathbf{1}\left(e_{t}^{N} \leq P_{t}^{s}\right)\right]+\lambda_{N} .
\end{aligned}
$$

Therefore, for part (a), if $D_{t}\left(p_{t}, \varepsilon_{t}\right)=\theta-b p+\varepsilon_{t}$ and $z=x-\theta+b p$, then the optimal $a_{t}^{i}, i<N$ satisfies

$$
\begin{aligned}
& \mathrm{E}_{P_{t}^{s}}\left[\mathbf { 1 } ( P _ { t } ^ { s } \geq e _ { t } ^ { i + 1 } ) \left(\int_{z-S_{t}^{i}+a^{i}}^{z-S^{i+1}+a^{i}}\left[-e_{t}^{i}+R_{t}^{\prime}\left(z-\xi+a^{i}\right) f(\xi)\right] d \xi\right.\right. \\
& \left.\left.+\left(e_{t}^{i+1}-e_{t}^{i}\right) \bar{F}\left(z-S_{t}^{i+1}+a^{i}\right)\right)\right] \\
& +\mathrm{E}_{P_{t}^{s}}\left[\mathbf { 1 } ( e _ { t } ^ { i } \leq P _ { t } ^ { s } < e _ { t } ^ { i + 1 } ) \left[\int_{z-S_{t}^{i}+a^{i}}^{z-\tilde{S}_{t}\left(P_{t}^{s}\right)+a^{i}}\left[-e_{t}^{i}+R_{t}^{\prime}\left(z-\xi+a^{i}\right) f(\xi)\right] d \xi\right.\right. \\
& \left.\left.+\left(P_{t}^{s}-e_{t}^{i}\right) \bar{F}\left(z-\tilde{S}_{t}\left(P_{t}^{s}\right)+a^{i}\right)\right]\right]+c_{t}^{i+1}-c_{t}^{i}+\lambda_{i}-\lambda_{i+1}=0,
\end{aligned}
$$

for $i=N, a_{t}^{N}$ is the solution of

$$
\begin{aligned}
& \mathrm{E}_{P_{t}^{s}}\left[\mathbf { 1 } ( e _ { t } ^ { N } \leq P _ { t } ^ { s } ) \left[\int_{z-S_{t}^{N}+a^{N}}^{z-\tilde{S}_{t}\left(P_{t}^{s}\right)+a^{N}}\left[-e_{t}^{N}+R_{t^{\prime}}\left(z-\xi+a^{N}, P_{t}^{s}\right) f(\xi)\right] d \xi\right.\right. \\
& \left.\left.+\left(P_{t}^{s}-e_{t}^{N}\right) \bar{F}\left(z-\tilde{S}_{t}\left(P_{t}^{s}\right)+a^{N}\right)\right]\right]+\lambda_{N}-c_{t}^{N}=0
\end{aligned}
$$

and $\lambda_{i}\left(a^{i}-a^{i-1}\right)=0, \lambda_{i} \geq 0$ for all $i=1, \ldots, N$. Part (b) can be shown analogously so we skip the details.

\section{Notes}

\footnotetext{
${ }^{1}$ (http:/ / www.cers.water.ca.gov/pdf-files/other-contracts / natural-gas-cntrcts/natural-as-backgrnd.pdf).
}

${ }^{2}$ The wholesale price contract can be thought of as a special case of option contracts, where the premium is the full price and the exercise price is zero.

${ }^{3}$ For any given $k$ and period $t=k L+1 \ldots,(k+1) L$, except $t=(k+1) L$, the threshold levels for exercising options will also depend on the initial inventory level $x$ at period $k L+1$.

\section{References}

Bartholomew, D. 2005. Spring. HP Reinvents, Slowly. CFO IT.

Chan, L. M. A., Z. J. Max Shen, D. Simchi-Levi, J. L. Swann. 2004. Coordination of pricing and inventory decisions: A survey and 
classification. D. Simchi-Levi, S. D. Wu, Z. J. M. Shen eds. Handbook of Quantitative Supply Chain Analysis: Modeling in the E-Business Era. Kluwer Academic Publishers, Norwell, MA, 335-392.

Chao, X., S. X. Zhou. 2006. Joint inventory-and-pricing strategy for a stochastic continuous-review system. IIE Trans. 38: 401-408.

Chen, F. Y., S. Ray, Y. Song. 2006. Optimal pricing and inventory control policy in periodic-review systems with fixed ordering cost and lost sales. Nav. Res. Log. 53: 117-136.

Chen, H., O. Q. Wu, D. D. Yao. 2010. On the benefit of inventory-based dynamic pricing strategies. Prod. Oper. Manag. 19(3): 249-260.

Chen, X., D. Simchi-Levi. 2004a. Coordinating inventory control and pricing strategies with random demand and fixed ordering cost: The finite horizon case. Oper. Res. 52: 887-896.

Chen, X., D. Simchi-Levi. 2004b. Coordinating inventory control and pricing strategies with random demand and fixed ordering cost: The infinite horizon case. Math. Oper. Res. 29: 698-723.

Chen, X., D. Simchi-Levi. 2006. Coordinating inventory control and pricing strategies: The continuous review model. Oper. Res. Lett. 34: 323-332.

Elmaghraby, W., P. Keskinocak. 2003. Dynamic pricing in the presence of inventory considerations: Research overview, current practices, and future directions. Manage. Sci. 49: 1287-1309.

Federgruen, A., A. Heching. 1999. Combined pricing and inventory control under uncertainty. Oper. Res. 47: 454-475.

Feng, Y., F. Y. Chen. 2003. Joint pricing and inventory control with setup costs and demand uncertainty. Working paper, Chinese University of Hong Kong.

Feng, Y., F. Y. Chen. 2007. Joint pricing and inventory control in periodic review systems: Optimality and optimization of (s, S, p) policies. Working paper, Chinese University of Hong Kong, Shatin, Hong Kong.

Fu, Q., C.-Y. Lee, C. P. Teo. 2010. Procurement risk management using options: Random spot price and the portfolio effect. IIE Trans. 42: 793-812.

Haksoz, C., S. Seshadri. 2007. Supply chain operations in the presence of spot market: A review with discussion. J. Oper. Res. Soc. 58: 1412-1429.

Heyman, D. P., M. J. Sobel. 1984. Stochastic Models in Operations Research. McGraw-Hill, New York.
Lundberg, D. G. 1984. Linear and Nonlinear Programming. AddisonWesley.

Martínez-de-Albéniz, V., D. Simchi-Levi. 2005. A portfolio approach to procurement contracts. Prod. Oper. Manag. 14: 90-114.

Milner, J. M., P. Kouvelis. 2007. Inventory, speculation, and sourcing strategies in the presence of online exchanges. MESOM 9(3): 312-331.

Nagali, V., J. Hwang, D. Sanghera, M. Gaskins, M. Pridgen, T. Thurston, P. Mackenroth, D. Branvold, P. Scholler, G. Shoemaker. 2008. Procurement risk management (PRM) at HewlettPackard company. Interfaces 38: 51-60.

Petruzzi, N. C., M. Dada. 1999. Pricing and the newsvendor model: A review with extensions. Oper. Res. 47: 183-194.

Polatoglu, H., I. Sahin. 2000. Optimal procurement policies under price-dependent demand. Int. J. Prod. Econ. 65: 141-171.

Schummer, J., R. V. Vohra. 2003. Auctions for procuring options. Oper. Res. 51(1): 41-51.

Thowsen, G. T. 1975. A dynamic, non-stationary inventory problem for a price/quantity setting firm. Nav. Res. Log. Quar. 22: 461476.

Topkis, D. M. 1998. Supermodularity and Complementarity. Princeton University Press, Princeton, NJ.

Whitin, T. M. 1955. Inventory control and price theory. Manage. Sci. 2: 61-68.

Wu, D. J., P. Kleindorfer. 2005. Competitive options, supply contracting and electronic markets. Manage. Sci. 51: 452-466.

Yano, C. A., S. M. Gilbert. 2005. Coordinated pricing and production/procurement decisions: A review. Chakravarty, A., J. Eliashbert eds. Managing Business Interfaces: Marketing, Engineering and Manufacturing Perspectives. Springer, New York.

Yazlali, O., F. Erhun. 2004. Managing demand uncertainty with dual supply contracts. Working paper, Stanford University.

Yi, J., A. Scheller-Wolf. 2003. Dual sourcing from a regular supplier and a spot market. Working paper, Carnegie Mellon University.

Zabel, E. 1972. Multi-period monopoly under uncertainty. J. Econ. Theory 5: 524-536.

Zhu, K., U. W. Thonemann. 2009. Coordination of pricing and inventory control across products. Nav. Res. Log. 56: 175-190. 